Dirk Olbers · Vladimir O. Ivchenko

\title{
On the meridional circulation and balance of momentum in the Southern Ocean of POP
}

Received: 26 June 2001 / Accepted: 2 November 2001

(C) Springer-Verlag 2001

\begin{abstract}
The circulation of the Southern Ocean is studied in the eddy-resolving model POP (Parallel Ocean Program) by an analysis of zonally integrated balances. The TEM formalism (Transformed Eulerian Mean) is extended to include topography and continental boundaries, thus deviations from a zonally integrated state involve transient and standing eddies. The meridional circulation is presented in terms of the Eulerian, eddy-induced, and residual streamfunctions. It is shown that the splitting of the meridional circulation into Ekman and geostrophic transports and the component induced by subgrid and Reynolds stresses is identical to a particular form of the zonally integrated balance of zonal momentum. In this balance, the eddy-induced streamfunctions represent the interfacial form stresses by transient and standing eddies and the residual streamfunction represents the acceleration of the zonal current by density fluxes in a zonally integrated frame. The latter acceleration term is directly related to the surface flux of density and interior fluxes due to the resolved and unresolved eddies. The eddy-induced circulation is extremely vigorous in POP. In the upper ocean a shallow circulation, reversed in comparison to the Deacon cell and mainly due to standing eddies, appears to the north of Drake Passage latitudes, and in the Drake Passage belt of latitudes a deep-reaching cell is induced by transient eddies. In the resulting residual circulation the Deacon cell is largely cancelled and the residual advection of the zonal mean potential density is balanced by diapycnal eddy and subgrid fluxes which are strong in the upper few hundred meters but small in the ocean interior. The balance of zonal momentum is consistent with other eddy-resolving models; a new aspect is the clear identification of density effects in the zonally integrated balance. We show that the wind stress
\end{abstract}

Responsible Editor: Jörg-Olaf Wolff

D. Olbers $(\varangle) \cdot$ V. O. Ivchenko

Alfred Wegener Institute for Polar and Marine Research,

Bremerhaven, Germany

e-mail: dolbers@awi-bremerhaven.de and the stress induced by the residual circulation drive the eastward current, whereas both eddy species result in a braking. Finally, we extend the Johnson-Bryden model of zonal transport to incorporate all relevant terms from the zonal momentum balance. It is shown that wind stress and induction by the residual circulation carry an eastward transport while bottom form stress and the stress induced by standing eddies yield westward components of transport.

Keywords Antarctic Circumpolar Current · Parallel Ocean Program · Transformed Eulerian Mean . Transient and standing eddies $\cdot$ Zonal momentum balance

\section{Introduction}

The air-sea exchange processes in the Southern Ocean exhibit a belt of a strong easterly wind stress over most of the area occupied by the Antarctic Circumpolar Current (ACC) and the Polar Front (PF) system, and heat loss to the atmosphere over the Antarctic Zone (which is the area between the PF and the Antarctic coast). Mechanical and thermohaline forcing of the ocean are interlinked in a complex and not yet fully understood way of frictional and water-mass conversion processes which establish one of the major currents of the world ocean. During the past two decades of oceanographic research - the WOCE area - many important contributions towards a description and a theoretical understanding of this current system have been made (a recent review is presented by Rintoul et al. 2001). Early interest was directed towards the dynamical balance of the flow, while most recent investigations seek the mechanisms which set the transport of the ACC. The current study contributes to both these issues.

The dynamical balance of the ACC addresses the relative role of terms in the balance of momentum. Here, zonal momentum was of most concern because the current is predominantly eastward and the most obvious 
form of momentum input was thought to be established by the strong eastward zonal wind stress. Less interest was attributed to the meridional component of momentum, which is basically in geostrophic balance. Some early confusion about different concepts of integrating (or averaging) the zonal balance seems to be settled: zonal vs. streamline averaging, whereby the former introduces standing eddies into the balance and the latter naturally puts more emphasis on the transient eddies (e.g., Marshall et al. 1993; Ivchenko et al. 1996), level vs. isopycnal layer integration where the Coriolis force in the former approach is replaced largely by the interfacial form stress divergence in the latter. A "canonical" form of balance has been established (e.g., Wolff et al. 1991; Marshall et al. 1993; Killworth and Nanneh 1994; Gille 1997; Stevens and Ivchenko 1997; Olbers 1998; Rintoul et al. 2001). For the vertically integrated total momentum (surface to bottom), the balance is between input of eastward momentum by wind stress and loss by bottom form stress. Other terms are negligible if eddies are resolved. In the water column, zonal momentum is transported downward by interfacial form stress due to transient and standing eddies but other terms also appear (unless thermodynamical processes are absent, as in quasigeostrophic models). The current study helps to identify and quantify thermohaline forcing mechanisms entering the zonal balance.

What determines the transport? The time and zonally averaged zonal balance does not involve the zonal speed of the current or the transport. Nevertheless, during spinup or time-dependent conditions the offset of this balance accelerates the zonal current and thus, in this very direct way, determines the transport. In fact, many recent studies of the transport issue (e.g., Olbers and Wübber 1991; Cai and Baines 1996; Gnanadesikan and Hallberg 2000; Gent et al. 2001) have used the spinup concept and applied numerical models to diagnose the dependence of the model ACC transport on the external forcing fields, by switching on and off - or enhancing or reducing - the surface fluxes of momentum and/or heat and salt in the entire domain or in subareas, thus searching for the most important mechanisms and geographical regions. Also, the dependence of transport on parameterizations of eddy effects in coarse models was investigated. Other investigations, employing a more theoretical than engineering approach, try to reduce the complex thermodynamics of large-scale geophysical fluid motion to a manageable subsystem, unavoidably neglecting processes and flow structures which might still be important for an ultimate theory of ACC transport. Attempts to turn the zonal balance into a diagnostic for transport involves the parameterization of transient eddy fluxes of density by a combined GreenStone and thermal wind approach (e.g., Johnson and Bryden 1989), or evaluating the bottom form stress in terms of topographically excited Rossby waves and implementing simple linear friction laws for momentum transfer through interfaces and bottom (e.g., Charney and DeVore 1979; Olbers and Völker 1996; Völker
1999). In the present study we review and extend the Johnson-Bryden model of ACC transport to include the entire field of relevant dynamic and thermodynamic processes.

Another important topic of Southern Ocean circulation - actually relevant to the entire ocean - is that of meridional overturning. The overturning streamfunction of the Eulerian mass flow is by now a standard diagnostic output of numerical ocean models. It became evident by a Lagrangian-oriented analysis (averaging along isopycnals instead of geopotentials) that the prominent Deacon cell in the Southern Ocean overturning does not represent pathways of water-masses (Döös and Webb 1994; Karoly et al. 1997; Marsh et al. 2000; Speer et al. 2000). Eddy-driven flow transports mass and tracers meridionally in cells of narrow vertical extent and compensates in part for the northward Ekman branch and deep geostrophic return flow associated with the bottom form stress. The linkage of this circulation with the interfacial form stress divergence in the balance of zonal momentum is extensively discussed in Rintoul et al. (2001). An adequate way of resembling Lagrangian motion in a zonally averaged form on geopotentials is that of TEM (Transformed Eulerian Mean) and residual circulation, introduced by Andrews and McIntyre in 1976 and described in Andrews et al. (1987). The equivalence of the residual circulation (defined in geopotential coordinates) and isentropically averaged circulation (a Lagrangian quantity) was illustrated by McIntosh and McDougall (1996). TEM is employed in this study to describe the meridional circulation in relation to the imposed thermohaline fluxes and water-mass conversion. Combined with the zonal momentum balance, it becomes evident that the residual circulation streamfunction in fact represents the forcing of the zonal flow by thermohaline effects.

Most ingredients from the physics of zonal currents used in the present study can be elucidated by a simple conceptual model. Consider the balance of zonal momentum, integrated zonally and over three layers (which may be stratified), separated by isopycnals. The upper layer of thickness, $\eta_{1}$, includes the Ekman layer, the intermediate layer at $z=-\eta_{2}$ lies above the highest topography in the Drake Passage belt (the range of latitudes which run through Drake Passage), and the lower one reaches from $z=-\left(\eta_{1}+\eta_{2}\right)$ to the ocean bottom at $z=-h$. Writing the depth and zonally integrated northward volume flux in each layer as $V_{i}, i=1,2,3$, the steady-state balance of momentum reads:

$$
\begin{aligned}
& -f \bar{V}_{1}=-\overline{\eta_{1}^{*} p_{1 x}^{*}}+\tau_{0}-R_{1} \\
& -f \bar{V}_{2}=\overline{\eta_{1}^{*} p_{1 x}^{*}}-\overline{\eta_{2}^{*} p_{2 x}^{*}}-R_{2} \\
& -f \bar{V}_{3}=\overline{\eta_{2}^{*} p_{2 x}^{*}}-\overline{h p_{b x}}-R_{3},
\end{aligned}
$$

where $p_{i}$ is the pressure in the respective layers, $p_{b}$ the bottom pressure, the overbar denotes time and zonal mean, the star denotes the deviation from this average (the starred quantities thus include transient and standing eddies), $\tau_{0}$ is the zonal wind stress, and $R_{i}$ the 
appropriate Reynolds stress divergence. The meridional circulation is characterized by the pattern of meridional transports $\bar{V}_{i}$ which, in this isopycnal framework, are of Lagrangian quality. The Eulerian parts of $\bar{V}_{i}$ consist of the wind-driven component $-\tau_{0} / f$ in the top layer and a geostrophic component in the bottom layer, associated with the bottom form stress $\overline{h p_{b x}}$. Since $\sum_{i} \bar{V}_{i}=0$ by mass balance, and assuming that $R_{i}$ can be neglected, these Eulerian parts of the meridional circulation balance, and the same argument states that the overall balance of zonal momentum is between the applied wind stress and the bottom form stress. If, in addition, the flow is adiabatic, then the meridional transport in each layer must vanish, $\bar{V}_{i}=0$, and we find that the interfacial form stress $\overline{\eta_{i}^{*} p_{i x}^{*}}$ is vertically constant and equal to $\tau_{0}$ (and the bottom form stress). If there is exchange of mass between the layers, implying conversion of watermasses, $\bar{V}_{i}$ equals the net exchange rate with the neighboring layers (integral of diapycnal transport divergence south of the respective latitude), which implies diabatic interior effects and surface fluxes of density and, at the same time, a nonzero vertical divergence of the interfacial form stress. The thermohaline forcing of the zonal flow is thus hidden in the Coriolis force, or equivalently, in the meridional overturning streamfunction.

Equating the zonal pressure gradient with the northward geostrophic velocity, $f v_{g}^{*}=p_{x}^{*}$, and the layer thickness fluctuation with temperature anomaly, $\eta^{*}=T^{*} / \bar{\Theta}_{z}$, we recover (for zero $\bar{V}_{i}$ and $R_{i}$ ) the JohnsonBryden relation (Johnson and Bryden 1989)

$f \frac{\overline{v^{*} T^{*}}}{\overline{\bar{\Theta}_{z}}}=\tau_{0}$,

according to which the northward eddy heat flux $\overline{v^{*} T^{*}}$, normalized by the potential temperature gradient $\Theta_{z}$, in the circumpolar belt of the ACC is of the size of the zonal wind stress $\tau_{0}$. This simple formula established one of the recently most celebrated models of ACC transport. Assuming that only transient eddies (denoted by a prime) contribute, and parameterizing the heat flux by a gradient form, $\overline{v^{\prime} T^{\prime}}=-\kappa T_{y}$, and using the thermal wind, $f u_{z}=g \alpha T_{y}$, the relation indeed becomes prognostic for the zonal shear of the current, thus relating the transport $\mathscr{T}$ (relative to some reference level) to the zonally averaged wind stress $\tau_{0}$. However, numerous attempts with numerical models, whether adiabatic or full PE, failed to verify the prediction of the square-root dependence, $\mathscr{T} \sim \tau_{0}^{1 / 2}$, resulting from a Green-Stone parameterization of the diffusivity $\kappa$. However, also the linear model with constant $\kappa$ or a cubic relation, $\mathscr{T} \sim \tau_{0}^{1 / 3}$ (see Rintoul et al. 2001) were without success. This is not surprising in view of the very restrictive range of applicability of Eq. (2): it is only valid for adiabatic conditions, only for the water column below the Ekman layer and above the highest topography in the circumpolar belt, and only if Reynolds stresses are small in the balance of zonal momentum. Furthermore, if the prime denotes the transient eddy component, as should be the case for downgradient parameterization of eddy fluxes, the transport model assumes that the effect of standing eddies in the transport of zonal momentum is negligible.

The next section presents the TEM approach to include topography (see also McIntosh and McDougall 1996). Data from the POP model (Parallel Ocean Program, Smith et al. 1992) are used in Section 3 to describe the zonally integrated circulation in an eddy-resolving solution, and Section 4 presents the balance of zonal momentum. Finally, in Section 5, we derive and apply a generalized Johnson-Bryden theory to examine the importance of the different mechanisms of zonal acceleration. Some analytical result from this theory are outlined in the Appendices.

\section{A zonally integrated formulation of TEM}

The isopycnal framework is fairly complicated in full PE models (note that, strictly speaking, isopycnal derivative, zonal integration, and time average do not commute) and balances like Eq. (1) are cumbersome to diagnose from level models (e.g., Killworth and Nanneh 1994). In this environment the TEM framework (transformed Eulerian mean, e.g., Andrews et al. 1987) and the concept of residual circulation are frequently invoked. The following derivation of TEM, appropriate for the ocean, where topography may block the zonal path, can be found in parts also in McIntosh and McDougall (1996). Define time-averaged and zonally integrated ${ }^{1}$ variables $\{\phi\}$ on level surfaces by the curly bracket operator and denote by $\phi^{*}=\phi-\{\phi\} / L$, with $L=L(y, z)$ being the length of the circumpolar path at depth $z$ and latitude ${ }^{2} y$. The deviation $\phi^{*}$ thus contains transient and standing eddies. The balance of zonal momentum becomes:

$-f\{v\}=\frac{\partial\{\tau\}}{\partial z}-\frac{\partial}{\partial z}\{u w\}-\frac{\partial}{\partial y}\{u v\}+\sum_{\text {ridges }} \delta \bar{p}_{b}$,

where the overbar is the time average. Lateral subgrid stresses are ignored for simplicity. The sum of the pressure differences is extended over all submarine ridges interrupting the integration path at depth $z$ (continents are here included). Each ridge or continent contributes the difference between the values on the eastern and the western side, i.e., $\delta p_{b}=p\left(x_{E}, y, z=-h\right)-p\left(x_{W}, y\right.$, $z=-h)$. The interfacial form stress divergence is introduced into Eq. (3) by splitting the Eulerian velocity into the Stokes-like - eddy-induced - part and the remaining residual circulation,

\footnotetext{
${ }^{1}$ These are more convenient than zonally averaged variables in the case of interruption by topography. For example, the zonal integral of a 3-D divergence turns into the corresponding 2-D divergence of the zonal integral, $\left\{\nabla_{3} \cdot \mathbf{F}_{3}\right\}=\nabla_{2} \cdot\left\{\mathbf{F}_{2}\right\}$ if the kinematic boundary holds for $\mathbf{F}_{3}$.

${ }^{2}$ We simplify equations by use of local Cartesian coordinates. All data evaluations of the POP model are performed in spherical coordinates.
} 


$$
\{v\}=v_{\text {res }}+\frac{\partial \psi_{\text {ed }}}{\partial z} \quad\{w\}=w_{\text {res }}-\frac{\partial \psi_{\text {ed }}}{\partial y},
$$

where $\psi_{\text {ed }}=\left\{v^{*} \sigma^{*}\right\} / \Sigma_{z}$ is the eddy-induced streamfunction, with $\Sigma=\{\sigma\} / L$ the zonal mean of the potential density $\sigma$ (referred to the surface and normalized by a constant reference density) and $\left\{v^{*} \sigma^{*}\right\}=\{v \sigma\}-\{v\} \Sigma$ the eddy flux of potential density. From mass balance it may be shown that the vector $\{v\}=-\partial \psi / \partial z$, $\{w\}=\partial \psi / \partial y$ has a streamfunction $\psi$ despite possible interruptions of the zonal path by topography. Thus, there is a streamfunction $\psi_{\text {res }}=\psi+\psi_{\text {ed }}$ of the residual circulation as well, and then Eq. (3) becomes:

$$
\begin{aligned}
& \mathscr{J}\left(\psi_{\text {res }}-\psi_{\text {ed }}, U\right)+f \frac{\partial}{\partial z}\left(\psi_{\text {res }}-\psi_{\text {ed }}\right) \\
& =\frac{\partial\{\tau\}}{\partial z}-\frac{\partial}{\partial z}\left\{u^{*} w^{*}\right\}-\frac{\partial}{\partial y}\left\{u^{*} v^{*}\right\}+\sum_{\text {ridges }} \delta \bar{p}_{b},
\end{aligned}
$$

where $\mathscr{J}$ is the $y, z$-Jacobian. Here, advective terms have been split into mean and Reynolds stress contributions, e.g., $\{u v\}=\{v\} U+\left\{u^{*} v^{*}\right\}$ with $U=\{u\} / L$, and the mean terms further expressed by the residual and eddyinduced streamfunctions. Defining the Eliassen-Palm vector $\mathbf{F}$ and the bottom form stress $\mathscr{F}(z)$ for the level interval from hilltop down to level $z$ in the valleys,

$$
\begin{aligned}
& \mathbf{F}=\left[-\left\{u^{*} v^{*}\right\}+\psi_{\text {ed }} U_{z},\left(f-U_{y}\right) \psi_{\text {ed }}-\left\{u^{*} w^{*}\right\}\right] \\
& \mathscr{F}(z)=\int_{z}^{0} \sum_{\text {ridges }} \delta \bar{p}_{b} \mathrm{~d} z=\sum_{\text {ridges }} \int_{x_{W}(z)}^{x_{E}(z)} \mathrm{d} x \bar{p}_{b} \frac{\partial h}{\partial x},
\end{aligned}
$$

the balance Eq. (5) can be expressed as residual advection and flux divergences of zonal momentum, driving the zonal mean current,

$$
\mathscr{J}\left(\psi_{\text {res }}, U\right)+\frac{\partial}{\partial z}\left(f \psi_{\text {res }}-\{\tau\}+\mathscr{F}\right)-\nabla \cdot \mathbf{F}=0 .
$$

Notice that $\mathscr{F}(z) \equiv 0$ for levels above highest topography. For a large-scale flow the Eliassen-Palm vector simplifies to $\mathbf{F}=\left(-\left\{u^{*} v^{*}\right\}, f \psi_{\mathrm{ed}}\right)$, and in the case of negligible contribution from the Reynolds terms and advection of zonal momentum by the residual circulation, Eq. (7) describes a balance of momentum which is entirely in terms of vertical fluxes: at each level the interfacial form stress due to the eddy field, $f \psi_{\text {ed }}$, is balanced by the flux due to subgrid motion, $\{\tau\}$, the flux carried by the residual circulation, $f \psi_{\text {res }}$, and the flux by the bottom pressure gradient, $\mathscr{F}$.

The residual streamfunction combines mean Eulerian and eddy-induced transports to the residual transport of potential density in the zonally integrated balance:

$\mathscr{J}\left(\psi_{\mathrm{res}}, \Sigma\right)=-\frac{\partial J}{\partial z}-\frac{\partial\left\{J^{y}\right\}}{\partial y}$.

To derive Eq. (8), the boundary condition on the threedimensional subgrid flux $\left(J^{x}, J^{y}, J^{z}\right)$ of $\sigma$ - being normal at the bottom - is imposed, as well as the kinematic boundary condition on the three-dimensional velocity ${ }^{1}$. Notice that eddies affect the zonal mean potential density only via $J^{\perp}=\left(\left\{v^{*} \sigma^{*}\right\} \Sigma_{y} / \Sigma_{z}+\left\{w^{*} \sigma^{*}\right\}\right)$, representing the (zonally integrated) eddy flux normal to (zonal mean) isopycnals. It combines with the vertical subgrid flux $J^{z}$ for diffusion and convection to $J=\left\{J^{z}\right\}+J^{\perp}$ and enters in the balance as a vertical divergence. With transient eddies being resolved and caring for horizontal or isopycnal mixing, the subgrid $J^{y}$ term may be abandoned.

The connection of $\psi_{\text {res }}$ with subgrid mixing processes and thermohaline surface forcing becomes obvious by regarding the potential density field $\Sigma(y, z)$ in Eq. (8) as given and solving for the residual streamfunction. The balance Eq. (8) may be put into the form:

$\frac{\partial \psi_{\text {res }}}{\partial s}=-\frac{1}{|\nabla \Sigma|} \frac{\partial J}{\partial z}$
Fig. 1 Sketch to show the coordinate system oriented at the isopycnal $\Sigma(y, z)=\Sigma$. Antarctica is to the left and $y=Y_{\mathrm{DP}}$ is in the Drake Passage belt

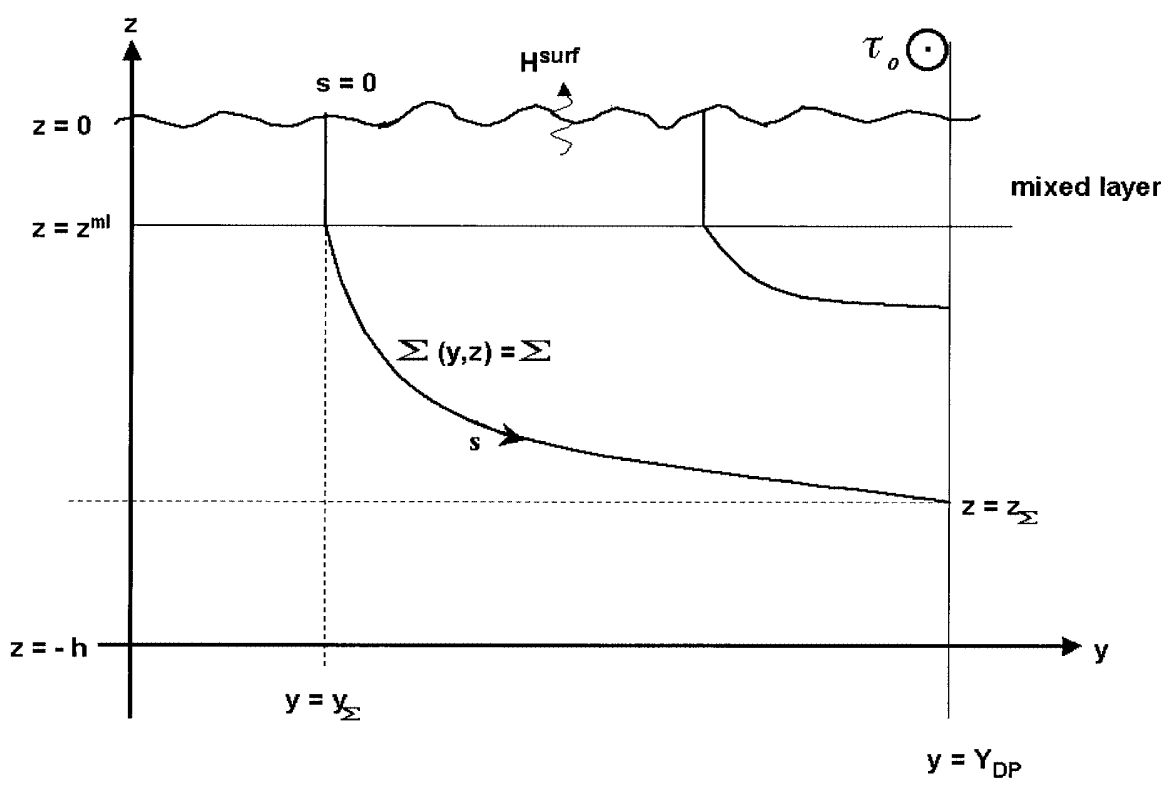


where the coordinate $s$ runs along isopycnals $\Sigma=\Sigma(y, z)=$ const (see Fig. 1 ). Suppose, for simplicity, that they outcrop at the surface $(s=0)$, run through a mixed layer where $\Sigma=\Sigma^{\mathrm{ml}}(y)$ is vertically constant, and then subduct into the interior. We may split the solution of Eq. (9) into a term from the surface flux boundary condition and one from the interior below the mixed layer, so in the coordinates $\left(y_{\Sigma}, s\right)$ we have

$\psi_{\text {res }}\left(y_{\Sigma}, s\right)=-\frac{\mathscr{H}_{0}\left(y_{\Sigma}\right)-J\left(y_{\Sigma}, z^{\mathrm{ml}}\right)}{\left|\Sigma_{y}^{\mathrm{ml}}\left(y_{\Sigma}\right)\right|}-\int_{s=z^{\mathrm{ml}}}^{s} \frac{\partial J / \partial z}{|\nabla \Sigma|} \mathrm{d} s^{\prime}$,

where $\left(y=y_{\Sigma}, s=0\right)$ is the outcrop point of the isopycnal $\Sigma$ at the surface and $z=z^{\mathrm{ml}}$ is the mixed-layer base at that latitude. The flux at the sea surface $\left\{J^{z}\right\}(y, s=0)=\mathscr{H}_{0}(y)$ is prescribed as boundary condition.

In the case that $J^{\perp}$ would vanish, the residual streamfunction $\psi_{\text {res }}$ is simply given by subgrid fluxes of density and could strictly be associated with diabatic thermohaline physics. Notice, however, even if eddies mix only along isopycnals and eddy-induced diapycnal fluxes are zero in a time mean framework (i.e., the transient eddy flux vector lies in the time mean isopycnal), this would not necessarily imply the vanishing of $J^{\perp}$. McIntosh and McDougall (1996), however, showed that the difference between geopotential zonal integration and isopycnal integration is of the order of the cube of the perturbation (eddy) amplitude. Thus, contributions to $J^{\perp}$ arising from locally isopycnic transient eddy flux are third-order, whereas contributions from locally diapycnic fluxes are second-order. Though, strictly speaking, only part of the residual streamfunction can clearly be attributed to diabatic physics, the part induced by locally isopycnic eddy mixing should be small. Hence, in this sense we may associate $J^{\perp}$ with diapycnic eddy fluxes and, consequently, $\psi_{\text {res }}$ with diabatic processes of water-mass conversion.

With $\psi=0$ at the surface and solid boundaries to ensure zero normal velocities there, we have also $\psi_{\text {res }}-\psi_{\text {ed }}=0$ there and thus, Eq. (7) integrates to:

$$
\begin{aligned}
& -f \psi=f\left(\psi_{\text {ed }}-\psi_{\text {res }}\right) \\
& \quad=f \frac{\left\{v^{*} b^{*}\right\}}{B_{z}}-f \psi_{\text {res }}=\left\{\tau_{0}\right\}-\{\tau\}-\mathscr{R}+\mathscr{F},
\end{aligned}
$$

which is the Johnson-Bryden relation in the most complete form. Here, $\mathscr{R}(z)$ collects small terms arising from the integral from top to level $z$ of the Reynolds stresses, the residual advection of $U$, and the $\psi_{\text {ed }} U$ terms. Apart from the lateral Reynolds term, the other terms in $\mathscr{R}$ will be neglected so that:

$\mathscr{R}(z) \approx \int_{z}^{0} \frac{\partial}{\partial y}\left\{u^{*} v^{*}\right\} \mathrm{d} z$.

By inserting Eq. (10), into Eq. (11) both external forcing functions - wind stress and surface density flux - are implemented in the balance of zonal momentum and the proportion of direct forcing by these agents becomes apparent. Of course, all other terms in the balance - the stratification in particular - depend indirectly on the external forcing, but this dependence cannot be revealed easily.

The eddy transport is further split into transient and standing components by setting, e.g., $v^{*}=\bar{v}^{*}+v^{\prime}$, the overbar being the time average. Then

$\left\{v^{*} \sigma^{*}\right\}=\left\langle\overline{v^{\prime} \sigma^{\prime}}\right\rangle+\left\langle\bar{v}^{*} \bar{\sigma}^{*}\right\rangle$,

and correspondingly for the Reynolds stress. The cornered brackets denote zonal integration. This implies a separation of the eddy-induced streamfunction into transient and standing contributions, $\psi_{\text {ed }}=\psi_{\text {trs }}+\psi_{\text {std }}$, and likewise for $\mathscr{R}$. Apparently, the density forcing of the zonal current is represented in the level framework by the residual streamfunction. Notice that Eq. (11) is valid not only at latitudes which are zonally unblocked by continents, but with the appropriate integration associated with the zonal integration operators $\{\cdots\},\langle\cdots\rangle$, it is also valid at latitudes which are interrupted by continents.

Taking Eq. (11) at the maximum depth, $h_{m}$, along the zonal path, the balance of total zonal momentum is obtained as:

$\left\{\tau_{0}\right\}-\left\{\tau_{b}\right\}+\mathscr{F}\left(-h_{m}\right)-\mathscr{R}\left(-h_{m}\right)=0$,

where $\tau_{b}$ is the frictional bottom stress and $\mathscr{F}\left(-h_{m}\right)$ the complete bottom form stress. The Reynolds part $\mathscr{R}$ is generally small, and $\tau_{b}$ can be neglected if the bottom topography is sufficiently high. Then, the zonal momentum is balanced by input due to wind stress and removal due to bottom form stress. This proved to be applicable in most models of the ACC (a summary of various models is found in Rintoul et al. 2001, see also below for the POP model).

\section{The zonally integrated circulation}

We have evaluated various fields from the output of a numerical model with eddy resolution, namely the POP model (Smith et al. 1992). The model and the particular experiment of this study are briefly described in Appendix A. The simulated ACC of POP is displayed in Fig. 2, showing the path of the current by the concentration of sea surface height isolines at the surface. As in other high-resolution models (e.g., The FRAM group 1991) and in the observed state of the ocean [from altimeter as, e.g., Le Traon et al. (1996), or from SST as, e.g., Olbers et al. (1992) or Hughes and Ash (2001)], the current is not a single zonal jet but a multiple jet system with a path which is strongly influenced by large-scale topographic features and the gap of Drake Passage, which it has to cross. Over long distances, particularly in the South Pacific, it follows the bathymetry. The gradual southward shifting of the current from the Atlantic throughout the Pacific is in accordance with 
Fig. 2 Sea surface height in the Southern Ocean of POP. $\mathrm{CI}=0.1 \mathrm{~m}$

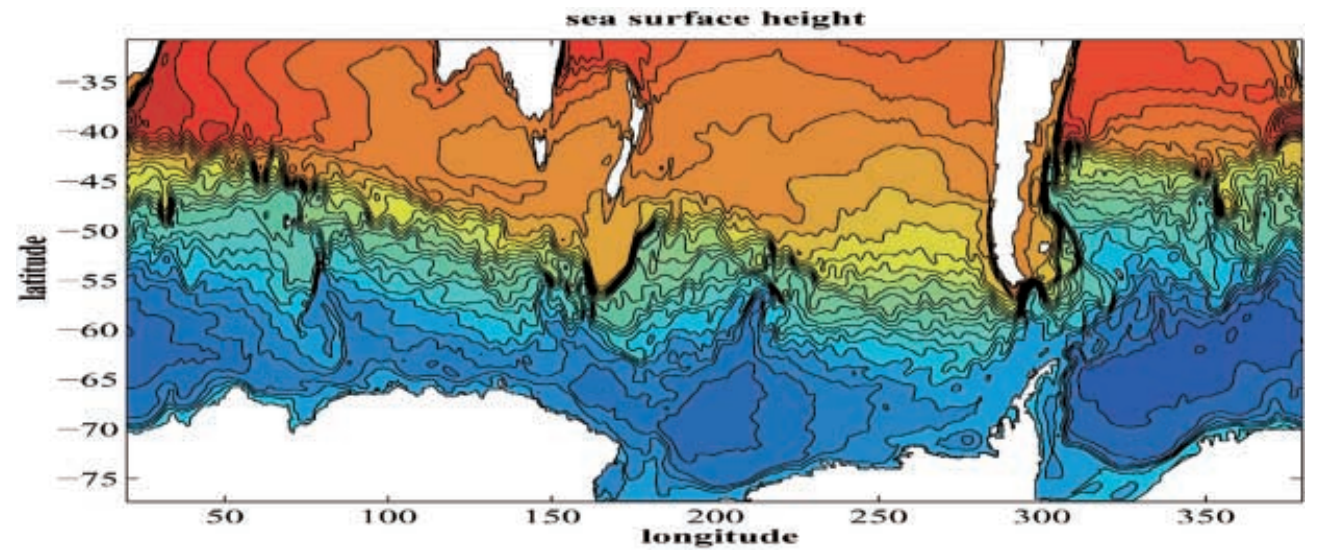

observations; it is interrupted by the sudden northward excursion in the lee of Drake Passage.

Obviously, in a zonal averaged picture many details of such a current system are lost and the single jets will be smeared out. Most important, the zonally integrated state in the Drake Passage band of longitudes will not represent the local structure of the current in Drake Passage. Thus, the transport of the averaged current in the Drake Passage band of latitudes is only about $50 \mathrm{~Sv}$, while the transport in this model through Drake Passage is $130 \mathrm{~Sv}$, very close to the observations (see Rintoul et al. 2001).

Most zonally integrated and time-averaged quantities that appear in the previous section have been extracted from the model output. We display them as function of depth and latitude. Values of the fields exist at a certain

Fig. 3 Zonally and time-averaged zonal mean velocity $U$, potential density field $\Sigma$, the Eulerian circulation streamfunction $\psi$, and the kinetic energy $E K E$ of transient eddies, as function of depth and latitude, taken from the POP model. Units: $U$ in $\mathrm{m} \mathrm{s}^{-1}, \psi$ in $\mathrm{Sv}, E K E$ in $10^{-2} \mathrm{~m}^{2} \mathrm{~s}^{-2}$

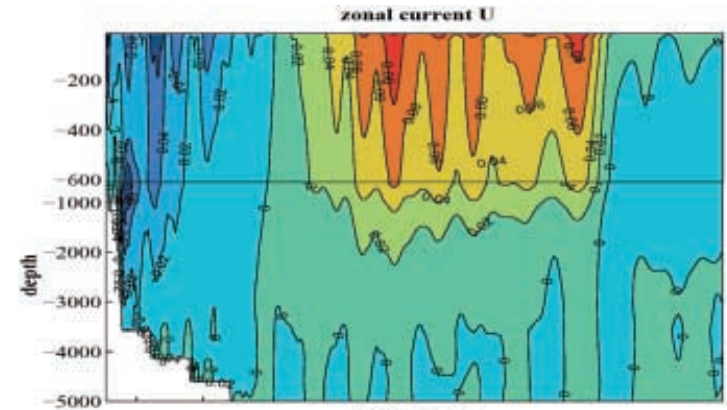

Ealerian $\psi$
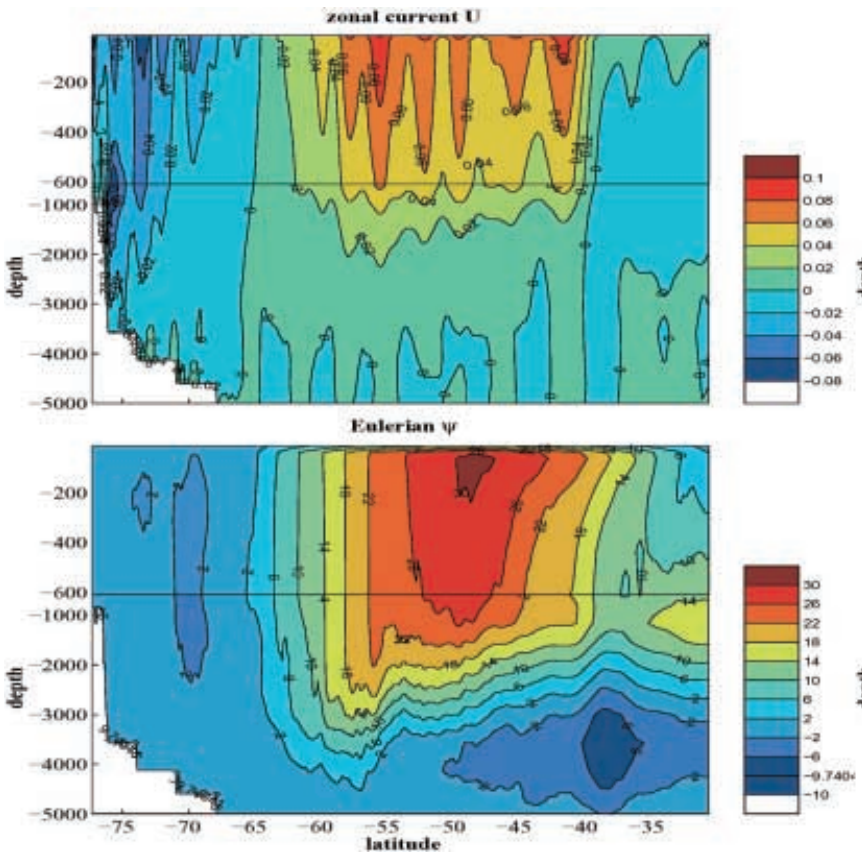

depth and latitude whenever there is such a point in the circumpolar domain that is wet, thus, e.g., in the upper unblocked area the integration is circumpolar (this is actually a small depth-latitude window concentrated in the belt spanning Drake Passage $-61.9^{\circ} \mathrm{S}$ to $56.0^{\circ} \mathrm{S}-$ down to depths of roughly $1500 \mathrm{~m}$ ), and in the vicinity of the bottom or the continental boundary in the south the integration may be only over a few wet grid points. Notice that $\psi_{\text {res }}=\psi+\psi_{\text {ed }}$, where $\psi$ can be obtained by vertical integration of $\{v\}$ with boundary condition $\psi(z=0)=0$, and $\psi_{\text {ed }}$ is defined by the meridional eddyinduced fluxes of potential density and the mean stratification. Unfortunately, the bottom pressure field is not available, and thus the quantity $\mathscr{F}$ could not be evaluated directly.

Figure 3 displays the basic Eulerian state of circulation of POP in the zonally integrated form. The zonal current in the zonal averaged picture still breaks up into clearly identifiable "jets"; two of them are in the Drake Passage belt, a further two are just north of Drake Passage latitudes and must arise from jet features in the Pacific and a

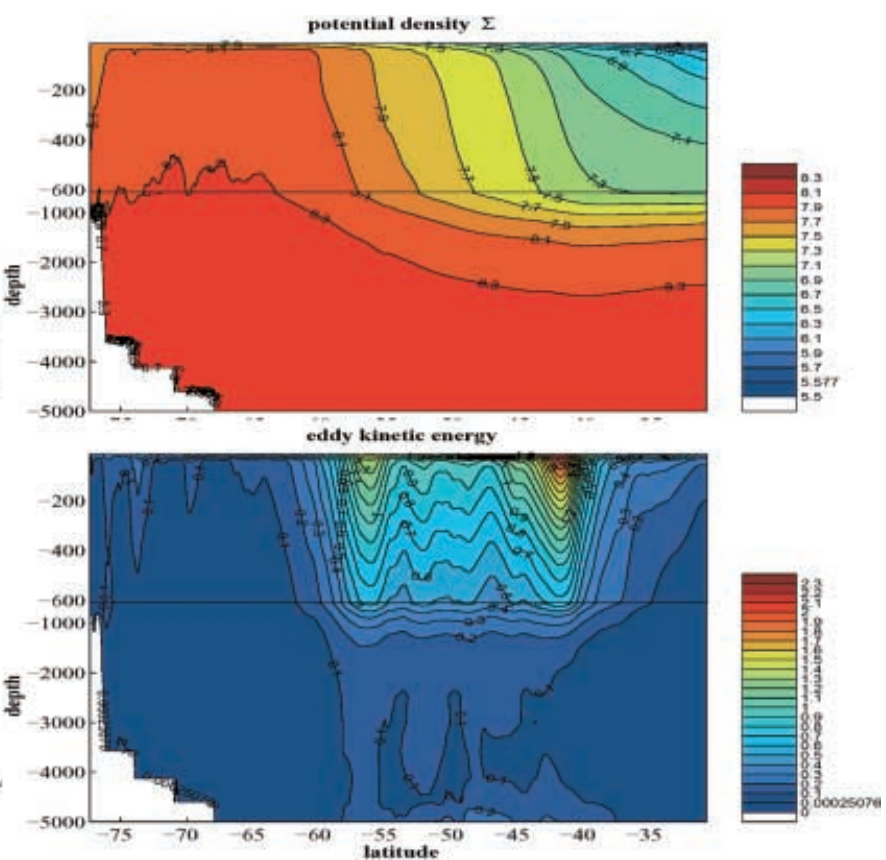


moderate eastward turning of the ACC off South America, and two more at about $45^{\circ} \mathrm{S}$ and $40^{\circ} \mathrm{S}$ are associated with the confluence zone of the Brazil Current and other jet-like features in the South Atlantic (see Fig. 2). The mean flow $U$, with surface velocities of roughly $0.1 \mathrm{~m} \mathrm{~s}^{-1}$, extends in eastward direction down to about $3000 \mathrm{~m}$. Close to the bottom there is weak westward flow; this also occurs in a meridionally banded structure in correlation with the jet bands at the top. The zonal mean potential density field $\Sigma$ shows the characteristic features of the Southern Ocean stratification: strong gradients in the surface layers (the mixed layer is not resolved), the strong downward slope of isopycnals from the surface to depths of roughly $2000 \mathrm{~m}$, starting at the Drake Passage belt latitudes, and a more or less homogeneous deep layer, extending to the surface in the Antarctic zone (the area south of the Drake Passage belt).

The meridional Eulerian transport, represented by $\psi$, is characterized by a fairly strong Deacon cell with a maximum of $27 \mathrm{~Sv}$ : the northward transport in the surface layer (the Ekman transport is mostly in the upper two layers, it has roughly $16 \mathrm{~Sv}$ in the Drake Passage belt) is returned - in the zonally integrated projection at great depths, mostly below $2000 \mathrm{~m}$. Two weak reverse and partially connected cells appear, one in the Antarctic zone and one below the Deacon cell; both are associated with bottom water flow. The structure and dynamics of the Deacon cell is extensively discussed in many studies of the Southern Ocean circulation (Döös and Webb 1994; Karoly et al. 1997, see also Rintoul et al. 2001). We only repeat here that it is a result of the zonal averaging. As can be seen from the zonally integrated momentum balance (Eq. 3), in the free (unblocked) water column, only ageostrophic meridional transports are possible (which are small because the Reynolds stress divergence is small), while at blocked depths geostrophically balanced transports can appear. In terms of local particle motion the northward flow induced by Ekman dynamics in the surface layer is indeed largely returned at only slightly deeper depths by eddy driving (the eddy-induced circulation, see below). In terms of zonally integrated mass transport, however, the return transport must be in the valleys of the blocking topography, or in the case of small or vanishing topography, by an Ekman bottom boundary flow. In POP it occurs by deep southward geostrophic currents. According to the balance of total momentum, (Eq. 14), the Eulerian terms of the meridional circulation balance if Reynolds terms are small: the northward Ekman transport $\tau_{0} / f$ is balanced by the geostrophic term $\mathscr{F} / f$ representing a southward return flow in the valleys.

Transient eddies result from the ACC jets by instability processes. POP resolves the eddy field in a realistic way with reasonable amplitudes (see the kinetic energy of the transient eddies in Fig. 3 and the discussion in Best et al. 1999) and thus, the mean Eulerian flow is backed by a significant eddy-induced field of transport. In a Lagrangian picture the eddy-induced transport of mass on isopycnals (which is the Stokes-like bolus transport) appears, which, together with residual transport, replaces the Eulerian transport. The division by $f$ turns the integrated balance of momentum, (Eq. 11), into a balance of meridional volume transports: indeed we can identify the level-mimic of eddy-induced isopycnal transport $\psi_{\text {ed }}$, the Ekman transport $\left\{\tau_{0}\right\} / f$, a frictional transport $\{\tau\} / f$ mainly associated with Ekman layer layers at top and bottom, the geostrophic transport in the valleys represented by $\mathscr{F} / f$, and the residual transport $\psi_{\text {res }}$. Informally expressed, these meridional volume transports are made responsible for "driving" the zonal transport of the ACC. Of course, it is the divergence of the corresponding stresses which accelerates the zonal mean current.

The eddy and the residual streamfunctions are shown in Fig. 4. These look very different from the Eulerian circulation: all have cells with reversed circulations as compared to the Deacon cell; imbedded are intense cells of significantly smaller sizes. The transient eddies induce a narrow, fairly deep-reaching, circulation of about $-10 \mathrm{~Sv}$ in the Drake Passage belt with two imbedded smaller cells, one in the upper $300 \mathrm{~m}$ of the water column (actually a double cell of maximum $-30 \mathrm{~Sv}$ ) and one in the depth ranges 1500 to $3500 \mathrm{~m}$ (with maximum $-25 \mathrm{~Sv}$ ). A smaller cell appears in the zone of northward ACC deflection north of Drake Passage and the confluence zone further to the north. Standing eddies generate an upper ocean intensive cell with maximum $-80 \mathrm{~Sv}$ in the deflection zone. The overwhelming importance of standing eddies in the eddy streamfunction and the residual circulation has been found in other eddy-resolving (or permitting) models; for FRAM (FRAM group 1991) this was shown in Karoly et al. (1997). All these cells combine in the total eddy streamfunction which presents a deep-reaching reverse eddy-induced flow of -10 to $-15 \mathrm{~Sv}$ with imbedded very strong localized cells in the upper ocean and at depth in the Drake Passage belt. In the resulting residual circulation the Deacon cell is replaced by a quite complicated pattern: the three small-scale reversed cells of the transient and standing eddy contributions appear with fairly strong and compact footprints; they are imbedded in a large-scale weaker flow which is northward in the surface layers (a remnant of the Ekman flow) and southward at great depths (a remnant of the Eulerian geostrophic return flow). Notice that the deep-reaching downward branch of the Deacon cell north of Drake Passage has completely vanished in $\psi_{\text {res }}$.

It is apparent from the circulation pattern of $\psi_{\text {res }}$ and the zonal mean stratification of potential density, shown in Fig. 3, that the intensely small-scale cells in the upper $600 \mathrm{~m}$ clearly transport zonal mean density across zonal mean isopycnals, but also the weaker deep flow is not entirely along isopycnals. We have evaluated some terms of the thermohaline balance (Eq. 8) (unfortunately, information about transport by subgrid processes and convection has not been stored). Figure 5 shows the Jacobian and the vertical divergence of the eddy-induced cross-isopycnal transport $J^{\perp}$ in the upper $600 \mathrm{~m}$ (below 

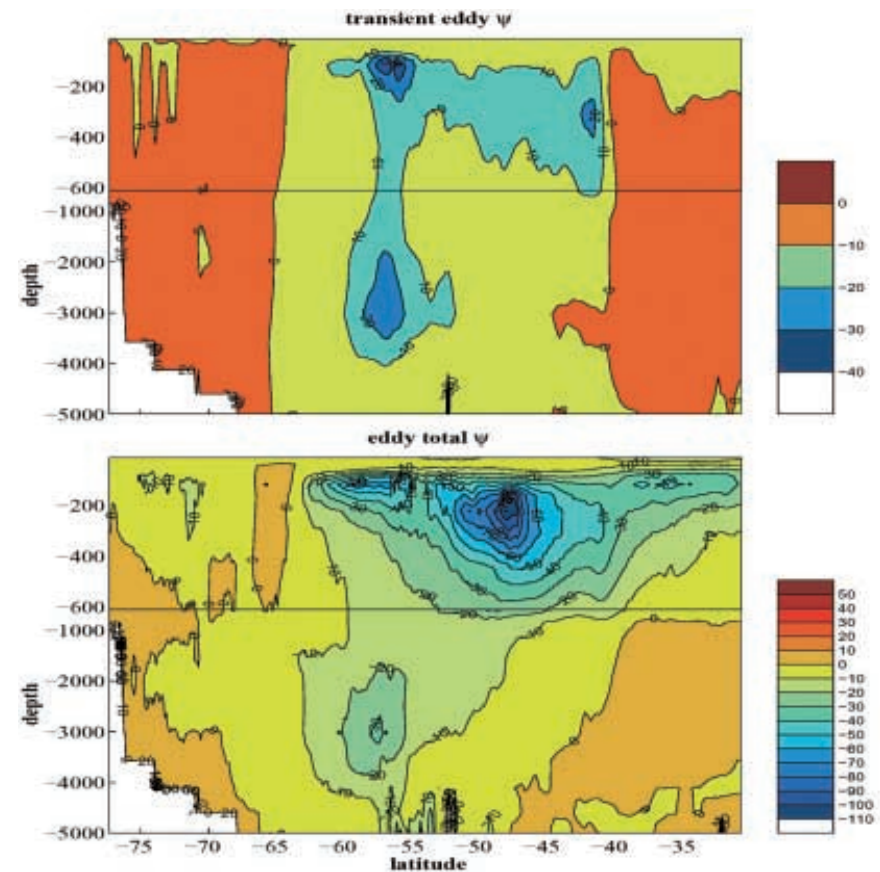

Fig. 4 Streamfunctions from the POP model as function of depth and latitude: transient eddy $\psi_{\text {trs }}$ (upper left panel), standing eddy $\psi_{\text {std }}$ (upper right panel), eddy total $\psi_{\text {ed }}$ (lower left panel), residual $\psi_{\text {res }}($ lower left panel). Unit: $\mathrm{Sv}, \mathrm{CI}=10 \mathrm{~Sv}$

the stratification is weak and both quantities are considerably smaller than in the upper ocean). The Jacobian indicates strong cross-isopycnal flow in the upper $300 \mathrm{~m}$, in and northward of the Drake Passage belt. The divergence of $J^{\perp}$ has a similar structure, being largely in balance with the advection, as revealed by the deficit of the balance shown in the third panel. Here, we notice the strong effects of surface processes, presumably by vertical subgrid diffusion and the surface exchange of density in the first layer. To the north of Drake Passage
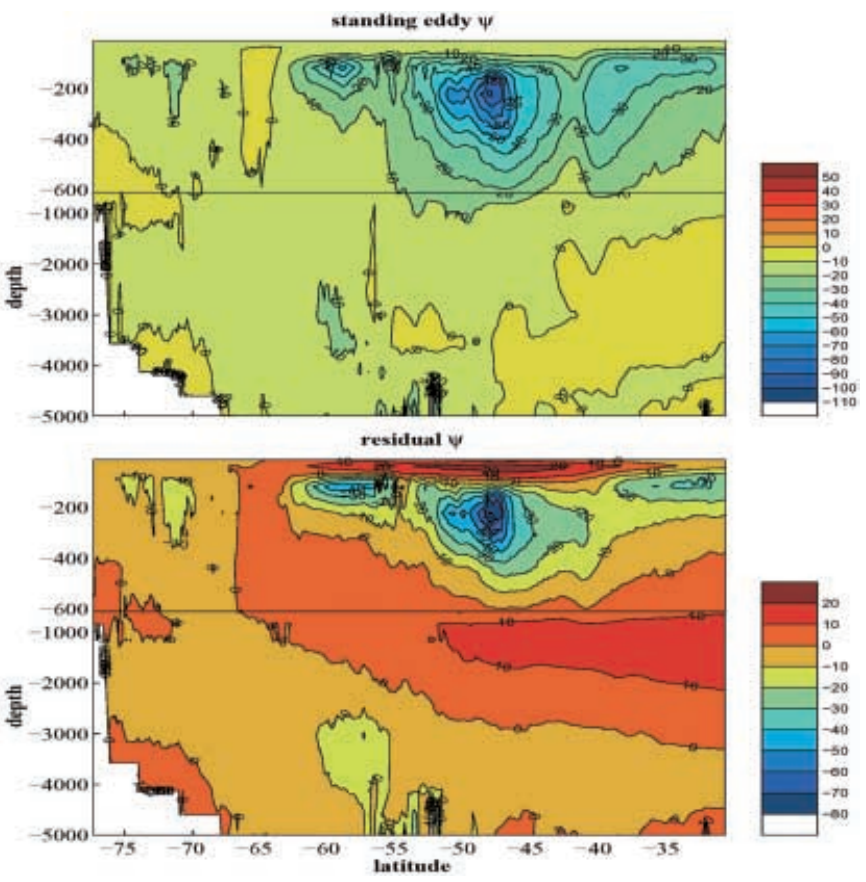

a subsurface maximum is visible. The attribution of the deficit to local diapycnal transports is, however, not evident, as pointed out by McIntosh and McDougall (1996), who showed that averaging the height of isopycnal on geopotentials is more appropriate than averaging potential density itself to detect diabatic processes.

\section{The balance of momentum}

As discussed in Section 5, the streamfunctions of the meridional circulation may be used to formulate the balance of zonal momentum. They enter as vertical divergence in the balance of the zonally integrated
Fig. 5 Display of terms of the potential density balance (Eq. 8) in the upper $600 \mathrm{~m}$. Upper panel Jacobian; middle panel divergence of $J^{\perp}$; lower panel deficit of these terms. Unit $10^{2} \mathrm{~m} \mathrm{~s}^{-1}$. $\mathrm{CI}=10$

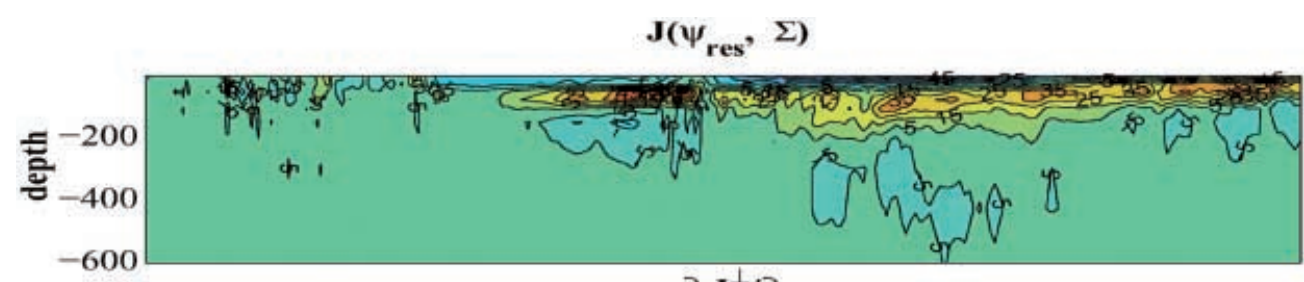

$-\partial \mathbf{J}^{\perp} / \mathbf{z}$
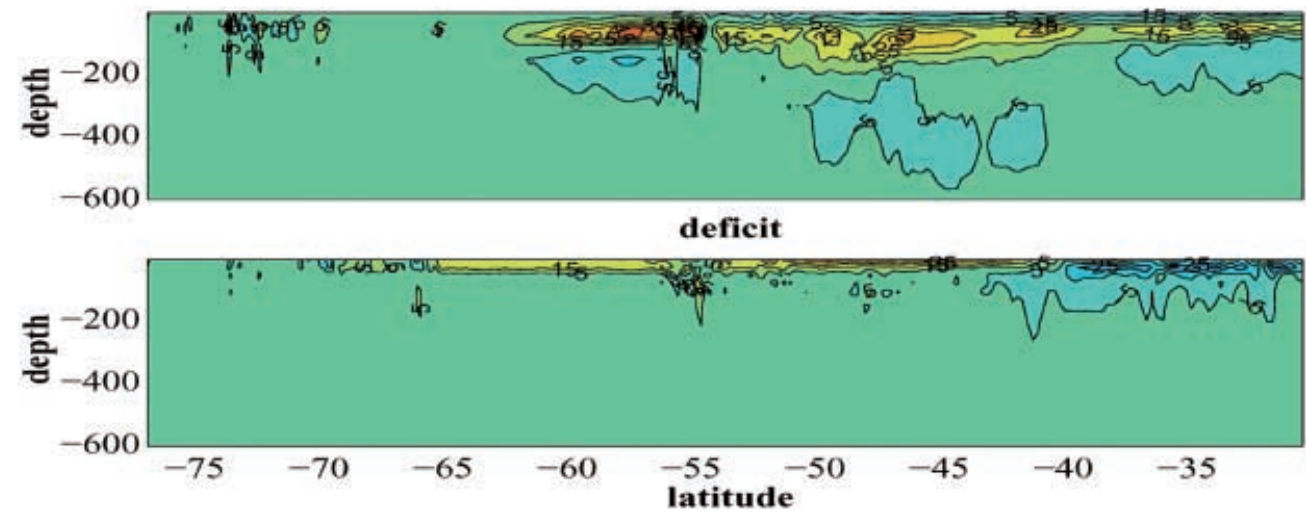
momentum or directly the particular form (Eq. 11) when integrated vertically. Most contributions to this balance of momentum can be estimated from the POP output. Those which have not been stored as model output (subgrid stress and bottom form stress) are lumped into $\mathscr{D}$, writing the balance then as

$-f \psi_{\text {trs }}-f \psi_{\text {std }}+f \psi_{\text {res }}+\left\{\tau_{0}\right\}-\mathscr{R}+\mathscr{D}=0$

$\mathscr{D}=-\{\tau\}+\mathscr{F}$.

The streamfunction terms of this balance and the Reynolds term integral are displayed in Fig. 6 for Drake Passage latitudes. The deficit $\mathscr{D}$ is shown as well for the Drake Passage belt, and in Fig. 7 (top panel) for latitudes from the Antarctic continent to $30^{\circ} \mathrm{S}$. The latter figure also displays the overall balance (Eq. 14) of zonal momentum between the wind stress and the bottom form stress (evaluated as deepest value of the

Fig. 6 Terms of the momentum balance in the Drake Passage belt. Upper panels Transient and standing eddy contribution; middle panels residual contribution and Coriolis force (which is the sum of the three streamfunction terms); lower panels Reynolds stress contribution and deficit (subgrid stress and bottom form stress). All quantities are normalized by $f_{0}=1.25 \times 10^{-4} \mathrm{~s}^{-1}$. Unit $\mathrm{Sv}, \mathrm{CI}=5 \mathrm{~Sv}$, except for Reynolds stress term: $\mathrm{CI}=0.5 \mathrm{~Sv}$

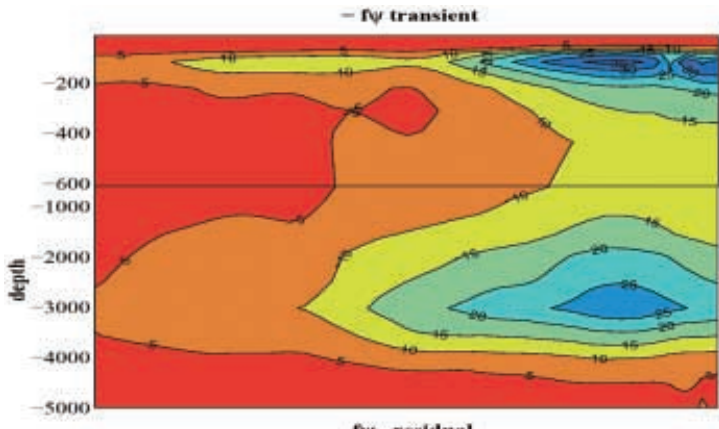

f\% rexidual
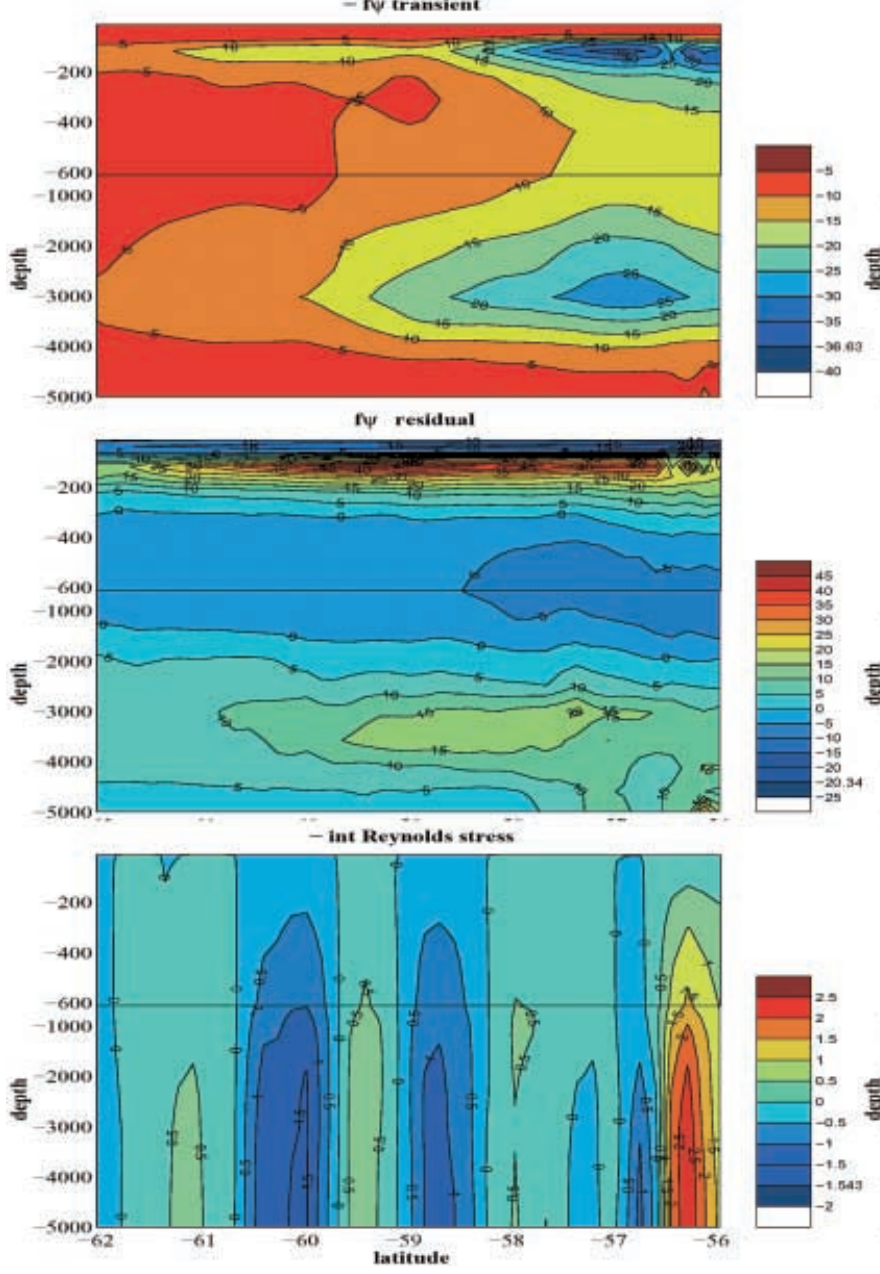

deficit) in the bottom panel, and the meridional integral of the terms in the balance (Eq. 15) over three different zones in the bottom panel $\left(80^{\circ} \mathrm{S}\right.$ to $62^{\circ} \mathrm{S}$ : Antarctic zone, $62^{\circ} \mathrm{S}$ to $56^{\circ} \mathrm{S}$ : Drake Passage belt, $56^{\circ} \mathrm{S}$ to $30^{\circ} \mathrm{S}$ : subtropical zone). All quantities are scaled by $f_{0}=1.25 \times 10^{-4} \mathrm{~s}^{-1}$ and thus have the dimension Sv. The $\tau_{0}$ term is vertically constant, increasing - in the scaled version - from $9.5 \mathrm{~Sv}$ at the southern latitude of Drake Passage, to $22.2 \mathrm{~Sv}$ at the northern latitude (see middle panel Fig. 7).

The presentation of the balance of zonal momentum in the form Eq. (15) may be rather unfamiliar; it is usually displayed and discussed in terms of the individual layers of an ocean model or in larger chunks of the vertical extent of the water column (say, the wind-mixed layer, the intermediate unblocked layer, and the deep blocked layer, see, e.g., the overview in Olbers 1998). Each term in Eq. (15), taken at a latitude $y$ and depth $z$, stands for the contribution of the acceleration, by a particular mechanism, of the surface to level $z$ and zonally integrated zonal momentum. The total acceleration is split here into the contributions of interfacial form stress by transient and standing eddies, by thermohaline forcing, by direct wind forcing, by Reynolds stress, by subgrid stress, and by bottom form stress, in the order in

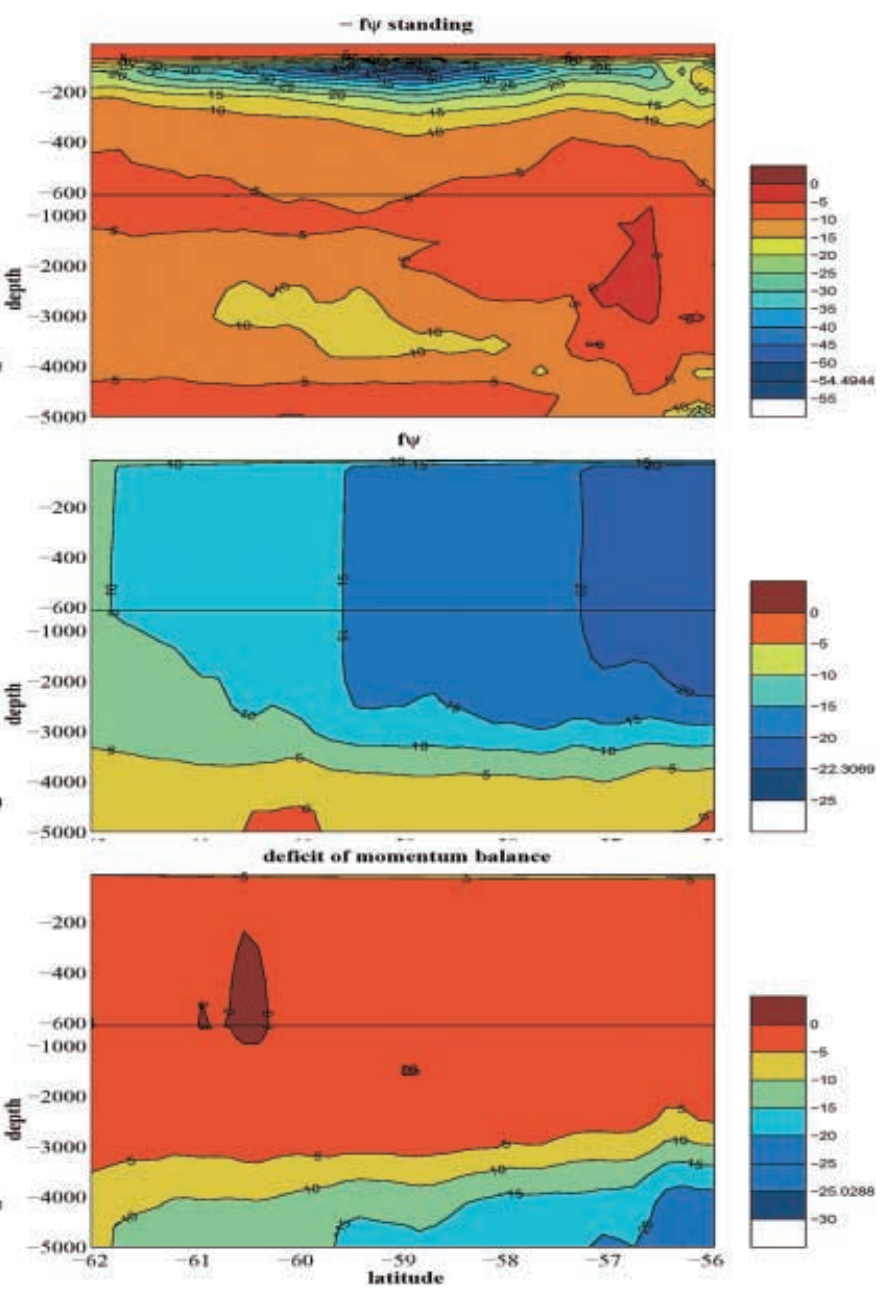


Fig. 7 Top panel Deficit term $\mathscr{D}$ of the momentum balance. For latitudes from the continent to $30^{\circ} \mathrm{S}$, normalized by

$f_{0}=1.25 \times 10^{-4} \mathrm{~s}^{-1}$. Unit $\mathrm{Sv}$, $\mathrm{CI}=5$ Sv. Bottom panel Vertically integrated momentum balance (also normalized by $\left.f_{0}=1.25 \times 10^{-4} \mathrm{~s}^{-1}\right)$, indicating a balance between wind stress and bottom form stress at each latitude (with small deviations due to Reynolds stress effects)

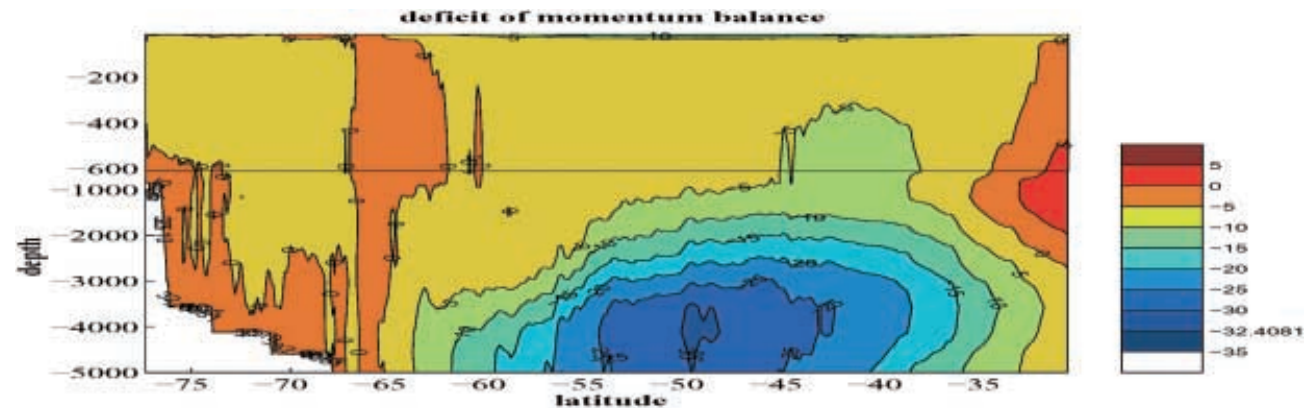

momentum balance

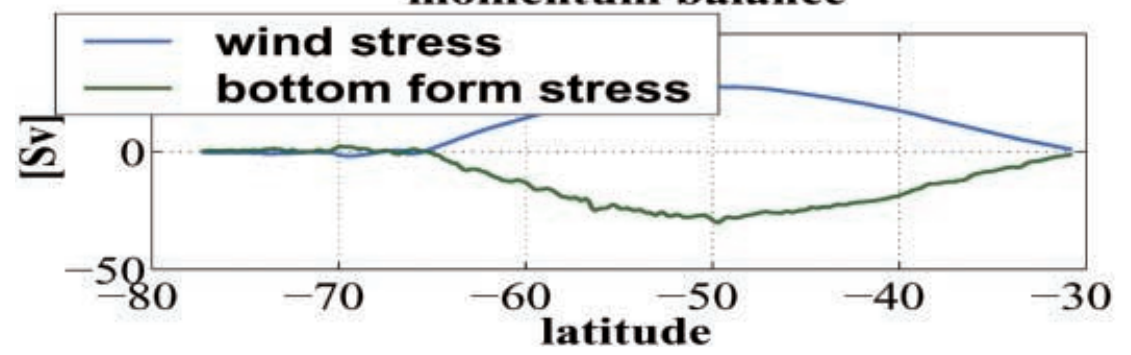

which they appear in Eq. (15). To obtain a view for the balance for a specific layer, one has to employ the difference - the vertical divergence - of Eq. (15) at the bounding interfaces.

Let us first consider the balance of the Drake Passage belt (Fig. 6 and middle panel of Fig. 8). The largest signal is obviously found in the contribution from the residual term, and the transient and standing eddies in the upper $500 \mathrm{~m}$, with a maximum at roughly $300 \mathrm{~m}$. In this depth range, residual forcing (by $\psi_{\text {res }}$ ) is accelerating the zonal current at a magnitude comparable, and in the peak even larger, than the wind stress. The eddies compensate this action, standing eddies being more efficient than the transient by a factor of roughly 2. Below $500 \mathrm{~m}$ (i.e., for the balance for the depth range from the surface to a depth of more than $500 \mathrm{~m}$ ), down to about $1000 \mathrm{~m}$ depth, these three terms drop well below the efficiency of the wind driving, but then regain strength in a second maximum around

Fig. 8 Zonally integrated balance for Antarctic zone (left), Drake Passage belt (middle), and subtropical zone (right). The bottom form stress contribution is also included in negative form as dashed curve to show that it meets the wind stress at the bottom, which presents the balance of total momentum at each latitude (middle panel). The abbreviations refer to the terms in Eq. (15), with rey to $\mathscr{R}$ and frm to D. Unit $10^{9} \mathrm{~m}^{3} \mathrm{~s}^{-2}$
$3000 \mathrm{~m}$ depth, now, however, with transient eddies dominating over standing eddies. At these deep levels, the bottom form stress builds up and decreases towards its most negative value at the deepest level. Here, the three streamfunction contributions are small and sum to zero at the bottom (because the Eulerian streamfunction conserves mass) and the overall balance (for the total water column) is between wind and form stress, as indicated by Eq. (14), with very small Reynolds stress effects. In fact, the latter is increasingly small everywhere. As shown in Fig. 6, lowest left panel, the Reynolds stress contributes in cells with alternating sign, coherent with the jet axes, with a magnitude which is by at least a factor of 10 smaller than the other terms in the balance. Integrated over some of these cells, as in Fig. 8, the Reynolds stress effect is entirely negligible.

For the areas north and south of the Drake Passage belt (see Fig. 8), the zonal balance differs considerably from the previous case. In the Antarctic zone, south of Drake Passage, only standing eddies and the residual term contribute, with residual forcing eastward and standing eddies retarding the east flow. North of Drake Passage, the wind stress increases by a factor of about 4 . It is mainly balanced by bottom form stress at depth here arising also from continental blocking - and a
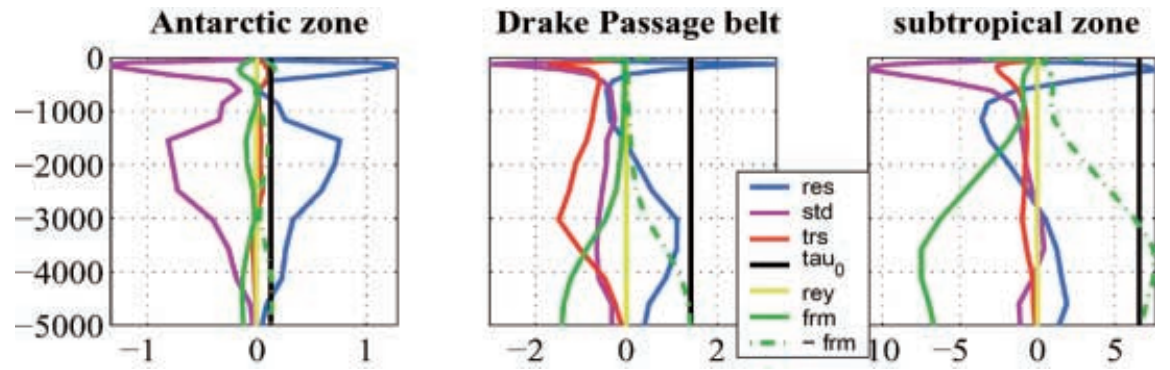
combination of all terms nearer to the surface (standing eddies are particularly prominent).

We have attributed all of the deficit $\mathscr{D}$ to the form stress $\mathscr{F}$. A closer look at the deficit pattern (lowest right panel of Fig. 6 and upper panel of Fig. 7) reveals a tiny surface boundary layer, contained in the upper layer in the model, where the influence of the subgrid stress $\{\tau\}$ is directly visible. We assume that it is small at greater depth, so the identification of $\mathscr{F}$ with the entire deficit $\mathscr{D}$ seems plausible.

Overall, there is no principle difference between the described balance of ACC momentum in POP and in other eddy-resolving models (see Stevens and Ivchenko 1997; Olbers 1998; Rintoul et al. 2001). The current presentation, by separating the Eulerian meridional circulation (or the integrated Coriolis force) into the eddyand density-driven parts, enables a clearer discussion of physical mechanisms. The focus on the balance between the Coriolis force, wind stress, Reynolds stress, and bottom form stress, usually extracted in these studies from the model output, is widened to elucidate the role of eddies and density forcing (water-mass conversion in a zonally integrated frame), which is otherwise revealed only in isopycnal analyses (e.g., Killworth and Nanneh 1994). In this view, it becomes obvious that eddies also act in level models by the interfacial form stress mechanism and that water-mass conversion represents an efficient agent for accelerating the zonal current.

\section{A generalized Johnson-Bryden theory}

In the TEM formalism, the zonally integrated balance of zonal momentum - in the form of Eqs. (5), (7) or (11) determines the residual streamfunction $\psi_{\text {res }}$, and the thermohaline balance Eq. (8) determines the potential density field $\Sigma$, given the eddy-induced transports. If the

Fig. 9 Left panels Three fits to parameterization of the transient eddy flux of potential density at latitude $59^{\circ} \mathrm{S}$ (left linear; middle GreenStone; right Visbeck et al.). For the fit, indicated by the straight line, only data below $500 \mathrm{~m}$ depth are used, these are circled. The axes are scaled. Right panel Diffusivity $\kappa(y)$ for the linear fit of the eddy flux to mean gradient (full line) and zonal current shear (dotted line). Unit $\mathrm{m}^{2} \mathrm{~s}^{-1}$ residual advection of momentum is retained, as in Eqs. (5) and (7), the TEM set must be supplemented by the balance of meridional momentum and the hydrostatic balance (see Andrews et al. 1987). As we apply the Johnson-Bryden concept, the roles of eddy and residual fields are reversed: we consider the zonal mean density field $\Sigma(y, z)$ as given and determine the residual streamfunction from the thermohaline balance (Eq. 8). Moreover, as in Johnson and Bryden (1989), the transient eddy flux of potential density is parameterized by a down-gradient form, $\left\langle\overline{v^{\prime} \sigma^{\prime}}\right\rangle=-\kappa L \Sigma_{y}$, and use of the thermal wind relation $f U_{z}=g \Sigma_{y}$ then turns (Eq. 11) into a prognostic equation for the shear $U_{z}$ of the zonal current (here we equate density with potential density). Thus, the parameterization and the balance of momentum imply

$$
\begin{aligned}
& \kappa L U_{z}=\frac{N^{2}}{f} \psi_{\mathrm{trs}} \\
& \frac{N^{2}}{f} \psi_{\mathrm{trs}}=\frac{N^{2}}{f^{2}}\left(\left\{\tau_{0}\right\}-\{\tau\}-\mathscr{R}+\mathscr{F}\right)+\frac{N^{2}}{f}\left(\psi_{\mathrm{res}}-\psi_{\mathrm{std}}\right)
\end{aligned}
$$

with a local Brunt-Väisälä frequency $N^{2}(y, z)=-g \Sigma_{z}$. Obviously, these equations allow the determination of the zonal current velocity and the associated transport relative to the bottom (or any other reference level) if the diffusivity and the terms on the rhs are known.

Following Green (1970) and Stone (1972), the form of the diffusivity is expressed as $\kappa=\alpha|f| \ell^{2} / \sqrt{\mathrm{Ri}}$, obtained for a baroclinically unstable flow, where $\mathrm{Ri}=N^{2} / U_{z}^{2}$ is the local Richardson number, $\ell$ is a measure of the eddy transfer scale, and the constant $\alpha$ measures the level of correlation between $v^{\prime}$ and $\sigma^{\prime}$ in the density flux, $\alpha=0.015 \pm 0.005$, according to Visbeck et al. (1997). These authors suggest that in the presence of differential rotation the eddy transfer may be restricted by the Rhines scale $\sqrt{U / \beta}$ rather than the Rossby radius, used in the conventional Green-Stone approach. In Fig. 9 we compare a linear model with vertically constant $\kappa$ with the nonlinear Green-Stone and Visbeck et al. concepts, using the POP data at latitude $59^{\circ} \mathrm{S}$ (left panels). The linear model is apparently most appropriate for the POP data. In the right panel of
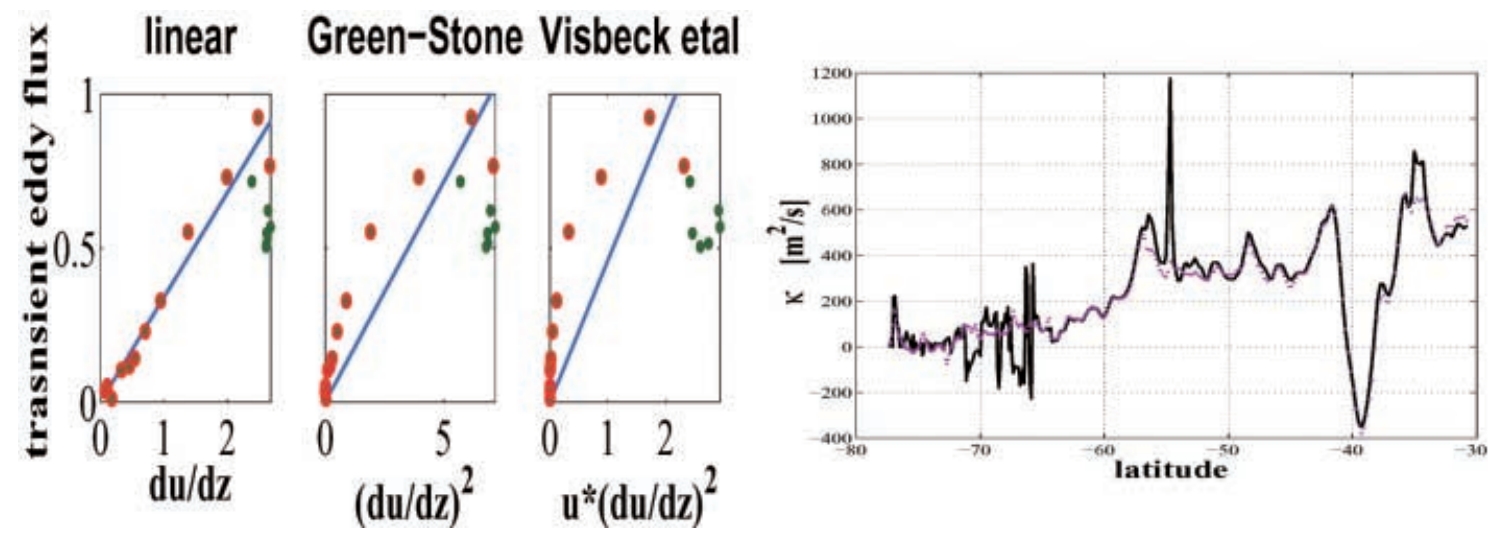
Fig. 9 a vertically constant diffusivity is applied at each latitude. This linear model obviously breaks down south of the Drake Passage belt and in the confluence zone where negative values of $\kappa$ result from the fit.

If the terms on the rhs of Eq. (17) and $\kappa$ are given, the equations yield by double vertical integration the transport of the zonal current relative to the bottom,

$$
\begin{aligned}
\mathscr{T}-\mathscr{T}_{b} & \\
= & -\frac{1}{\kappa} \int_{-h}^{0} \mathrm{~d} z \frac{z N^{2}}{L f^{2}}\left[\left(\left\{\tau_{0}\right\}-\{\tau\}-\mathscr{R}+\mathscr{F}\right)+f\left(\psi_{\text {res }}-\psi_{\text {std }}\right)\right],
\end{aligned}
$$

where $\mathscr{T}_{b}=h U(-h)$ and $L=L(y, z)$ should be used. The complete transport of the zonal mean current is thus expressed as the sum of seven terms,

$$
\mathscr{T}=\mathscr{T}_{b}+\mathscr{T}_{0}+\mathscr{T}_{\text {fr }}+\mathscr{T}_{\text {rey }}+\mathscr{T}_{\text {frm }}+\mathscr{T}_{\text {res }}+\mathscr{T}_{\text {std }} .
$$

Here, $\mathscr{T}_{\text {fr }}$ represents the contribution from the subgrid stress $\tau$ in the water column, and $\mathscr{T}_{\text {frm }}$ results from the bottom form stress. As outlined in Appendix B, the residual streamfunction term can further be evaluated to give a contribution which is directly proportional to the surface density flux (a weighted integral of the fluxes south of the Drake Passage belt, see Appendix B) and other contributions arising from interior diabatic subgrid fluxes of density as well as from the resolved eddy flux $J^{\perp}$. In the following, this separation is not used; $\psi_{\text {res }}$ data are taken directly. All transport terms in Eq. (19) are functions of latitude and the volume transport of the zonal mean ACC is then obtained by integration across the Drake Passage latitudes.
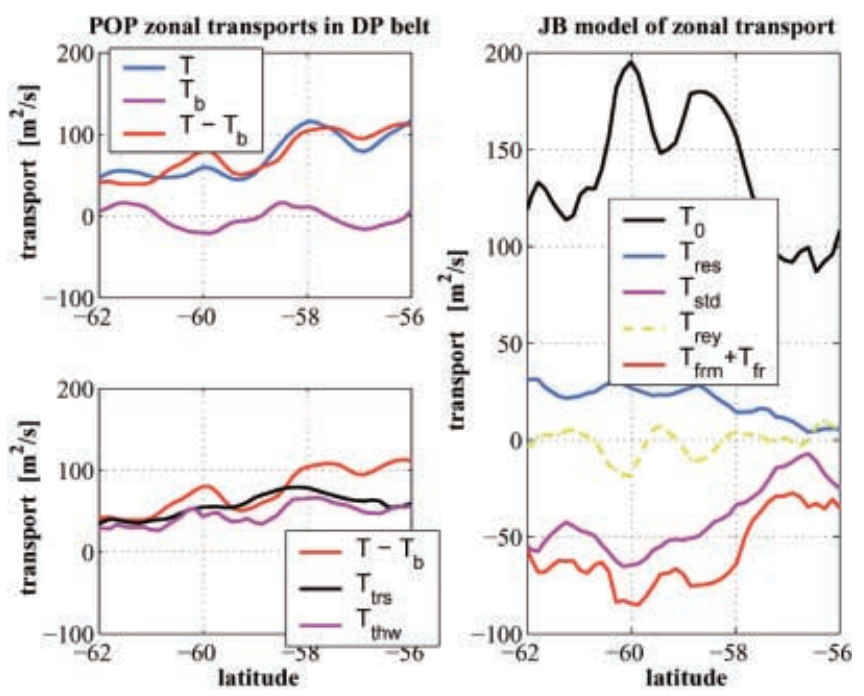

Fig. 10 Left panel Mean zonal transport in the Drake Passage belt (total transport full); relative to bottom dashed; bottom term dashdotted); right panel estimate of various transport terms in the separation Eqs. (19)
Most of the terms in Eq. (18) are readily evaluated from experiments with numerical circulation models. Figure 10 shows the $\mathscr{T}$ terms from POP data. Here, $\mathscr{T}$ and $\mathscr{T}_{b}$ use the simulated profile $U(z)$ of the zonal mean current. The upper left panel also shows the baroclinic transport $\mathscr{T}-\mathscr{T}_{b}$. In the lower left panel we show besides $\mathscr{T}-\mathscr{T}_{b}$ two other estimates of the baroclinic transport, one $\left(\mathscr{T}_{\text {trs }}\right)$ obtained by integrating Eq. (16) twice, with $\psi_{\text {trs }}$ from the model data, and one $\left(\mathscr{T}_{\text {thw }}\right)$ obtained from the thermal wind relation. For a perfect flux parameterization and perfect geostrophy, these three curves should collapse. It is revealed here that the constant- $\kappa$ fitting procedure, using only data from below $500 \mathrm{~m}$, is incapable of reproducing the upper level fluxes (see Fig. 9). This leads to a discrepancy between $\mathscr{T}_{\text {thw }}$, $\mathscr{T}$ trs and $\mathscr{T}-\mathscr{T}_{b}$. The other $\mathscr{T}$ terms are obtained using the above expression (18) and data of the applied wind stress, meridional eddy fluxes, the momentum deficit term $\mathscr{D}$, and the stratification. They are displayed in the right panel of Fig. 10. The integrated values of individual transport terms for the Drake Passage belt are listed in Table 1.

A few outstanding signatures are readily extracted from Fig. 10 and Table 1. It is obvious that the part of transport relative to bottom, $\mathscr{T}-\mathscr{T}_{b}$, carries most of the transport: although the bottom transport is locally a $20 \%$ contribution, its alternating sign yields a very low value in the integral. The total transport of the zonal mean velocity profile in the Drake Passage belt - with about $50 \mathrm{~Sv}$ - is significantly smaller than the transport through Drake Passage (about $130 \mathrm{~Sv}$ in POP). The reason is found in the nonzonal path of the ACC, which is mostly north of the latitudes spanned by Drake Passage (see Fig. 2).

With respect to forcing mechanisms, the direct wind forcing $\left(\mathscr{T}_{0}\right)$ is clearly dominant; together with the residual forcing $\left(\mathscr{T}_{\text {res }}\right)$ it establishes eastward flow at a rough proportion of wind to residual forcing of 6.5 . Remember that this number characterizes the directly forced contributions to the transport. Retarding mechanisms, yielding westward transports, are the bottom form-stress component $\mathscr{T}_{\text {frm }}$ and the standing eddies $\mathscr{T}_{\text {std }}$, the first leading slightly in magnitude. The

Table 1 Various transport terms integrated over the Drake Passage belt. The right column gives the percentage of the terms in the transport balance in relation to the transient eddy term

\begin{tabular}{lcc}
\hline Transport term & $\begin{array}{l}\text { Integral for } \\
\text { Drake Passage belt (Sv) }\end{array}$ & $\begin{array}{l}\text { Percentage } \\
\text { of balance }\end{array}$ \\
\hline $\mathscr{T}$ & 50.8 & \\
$\mathscr{T}_{b}$ & -1.2 & \\
$\mathscr{T}-\mathscr{T}_{b}$ & 52.0 & \\
$\mathscr{T}_{\text {thw }}$ & 31.5 & 100 \\
$\mathscr{T}_{\text {trs }}$ & 39.5 & 236 \\
$\mathscr{T}_{0}$ & 93.1 & -1 \\
$\mathscr{T}_{\text {rey }}$ & -0.7 & -99 \\
$\mathscr{T}_{\text {frm }}$ & -38.7 & 36 \\
$\mathscr{T}_{\text {res }}$ & 13.9 & -72 \\
$\mathscr{T}_{\text {std }}$ & -28.2 & \\
$\mathscr{T}$ & &
\end{tabular}


Reynolds stress forcing has alternating sign and is insignificant.

\section{Summary and conclusions}

We have applied the TEM formalism (Andrews et al. 1987) to data from the global ocean circulation model POP (Smith et al. 1992) to describe the zonal and meridional circulation in the model Southern Ocean in a zonally integrated frame, and to analyze the balances of potential density and zonal momentum. Fluxes of potential density by transient and standing eddies are used to express the Eulerian streamfunction of the meridional circulation by the eddy-induced and the residual streamfunctions. It is shown that the residual streamfunction at latitudes of the ACC is determined by the surface density flux and the subgrid and eddy-induced diapycnal fluxes (across the zonal mean isopycnals) to the south of the Drake Passage belt of latitudes. It is via the residual streamfunction that thermohaline processes induce an acceleration of the zonal current. Other terms in the zonal momentum balance arise from forcing by wind stress, from subgrid and Reynolds stresses, and from bottom form stress. The balance of zonal momentum is identical with the balance of the meridional mass transport when written in terms of the residual circulation, Ekman transport, eddy-induced transports (by both eddy species), deep geostrophic transport supported by pressure gradients in submarine valleys, and the contributions driven by Reynolds and subgrid stresses.

Eddies drive a quite vigorous circulation in POP, partially compensating the Ekman transport in the surface layer, but exceeding it substantially below the Ekman layer in the upper few hundred meters in the Drake Passage belt and further north in the confluence zone of the ACC and the Brazil current. Transient eddies contribute with an intense deep-reaching cell in the Drake Passage belt, and both streamfunctions add up to a residual circulation which clearly indicates diabatic processes in the upper few hundred meters, but is approximately adiabatic below. In this circulation the Deacon cell is no longer visible.

The balance of zonal momentum in POP follows the canonical scheme as described in many previous analyses of eddy-resolving models (see e.g., the summary in Olbers 1998). Wind stress is balanced by bottom form stress in the vertically integrated form of the balance, and this occurs latitude by latitude because lateral transport of momentum by Reynolds stress is negligible. Acceleration of the zonal current by eddy and thermohaline processes appears in the momentum balance as vertical divergence of momentum transports, represented by the eddy-induced and the residual streamfunctions. They have opposing influence: the stress induced by transient and standing eddies retards the eastward flow and the density-induced stress forces it. Bottom form stress is a major sink of eastward momentum below about $1000 \mathrm{~m}$ depth and at latitudes of the Drake Passage, and even stronger north of Drake Passage (where most of the stronger jets of the ACC are situated in POP).

A final question addressed in this study is that of the size of the mean zonal transport. We should distinguish between the transport through Drake Passage (which is $130 \mathrm{~Sv}$ in POP) and the transport associated with zonal mean current profile in the Drake Passage belt of latitudes (which is about $50 \mathrm{~Sv}$ in POP, showing that much of the ACC is missing in the zonal average). We have proposed an extension of the Johnson-Bryden model of the transport of the zonal mean current. It is found that a linear relationship between transient eddy potential density transport and meridional mean gradient is superior to nonlinear laws [thus the square-root dependence of transport on wind stress, proposed by Johnson and Bryden (1989), would not apply even if the simplified balance (Eq. 2) were appropriate]. The extended transport formula then combines the zonal acceleration mechanisms in a linear way and includes - besides driving by transient eddies and wind stress - also (in the order of their importance in POP) deceleration by bottom form stress and the stress induced by standing eddies, and acceleration by the thermohaline-induced stress. For the state of the POP experiment used in the present study, wind stress is by far the most important external force in generating the transport.

Though the external forcing parameters - wind stress and the surface density flux - appear in a linear way in the final transport formula, this does not reflect the dependence of the transport with respect to these parameters: there are other internal parameters in this formula which establish their values during the spinup of the circulation and thus might depend indirectly on the external forcing. These are the stratification of the zonal mean potential density (the Brunt-Väisälä frequency), the standing eddy density flux, and the interior eddy and subgrid density fluxes. Insight into the dependence of the time mean transport on the external forcing fields cannot be gained by a single model experiment. Of course, any attempt at a suite of experiments as, e.g., collected by Gent et al. (2001) with coarse resolution models, with an eddy-resolving model such as POP is denied by limited computer resources (remember that the present experiment was run over three decades only and is thus not in a complete thermally equilibrated state).

Acknowledgements We thank R. Malone and R. Smith for providing the POP data. Trevor McDougall and Chris Hughes helped to improve the manuscript with their critical comments, which are gratefully acknowledged.

\section{Appendix A: The POP model}

POP is a primitive equation high-resolution GCM (Smith et al 1992). The model uses a Mercator grid, allowing higher resolution in polar regions $\left(6.5 \mathrm{~km}\right.$ at $\left.78^{\circ}\right)$ compared to equatorial 


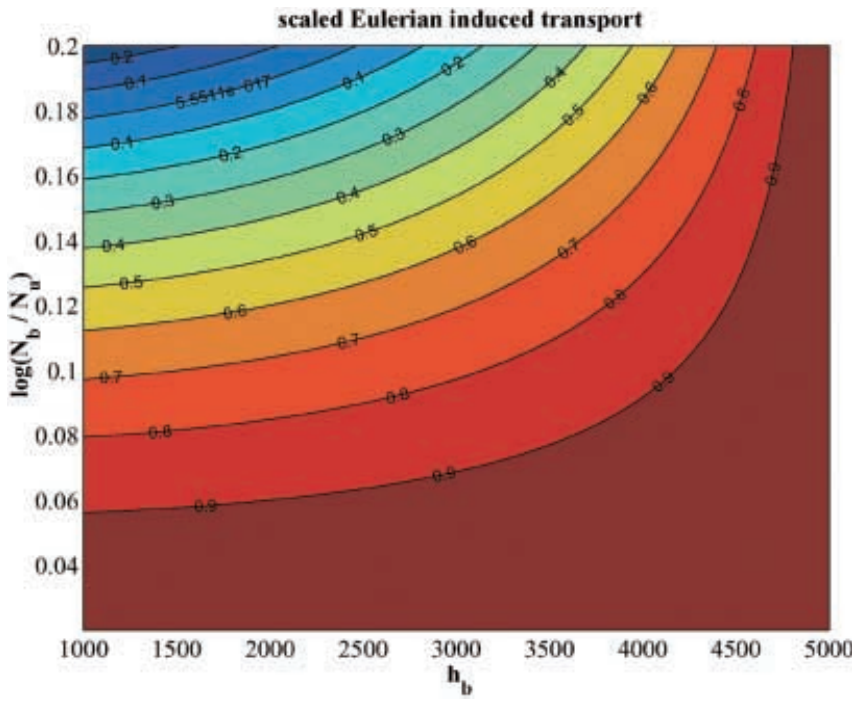

Fig. 11 The transport ratio $\left(\mathscr{T}_{0}+\mathscr{T}_{\text {frm }}\right) / \mathscr{T}_{0}$ as function of the form-stress scale parameter $h_{b}$ and $N_{b} / N_{0}$

regions (31.25 $\mathrm{km}$ at the equator). The vertical grid varies between $25 \mathrm{~m}$ at the surface and $550 \mathrm{~m}$ in the deep ocean (altogether 20 levels). The POP model is a Bryan-Cox type of model with an implicit free surface formulation of the barotropic mode. The numerical scheme does not need any smoothing of bottom topography for computational stability (Smith et al. 1992), which significantly lowers the ACC transport (Best et al. 1999), making it much more realistic than previous GCM with high resolution.

Surface heat flux is based on Barnier et al. (1995). However, in the case of ice being present, the sea-surface temperature is restored to $-2{ }^{\circ} \mathrm{C}$ with a 1 -month time scale. The surface salinity is restored to the monthly Levitus climatology (Levitus 1982). In the high latitudes (poleward of $70^{\circ}$ ) the temperature and salinity are relaxed to the annual Levitus climatology in the top $2 \mathrm{~km}$. The output used in this study is a 5 years' time average of 10-year simulations, taken from the last of three runs using the 1985-1994 ECMWF winds, each run initialized from the final state of the previous experiment. A 3-day average of winds was used. Further details of the POP model and experiments are given by Maltrud et al. (1998).

\section{Appendix B: Some analytical results for the J-B model}

We turn our attention to some of the mechanisms in Eq. (18), which can be investigated further by analytical means.

\section{Eulerian mean terms}

The contribution from the Eulerian mean terms, deriving from $\tau_{0}$ and $\mathscr{F}$, involve Ekman and geostrophic transports. Here, the abbreviation $\tau_{0}=\left\{\tau_{0}\right\} / L$ is used. They can be expressed as:

$\mathscr{T}_{0}=\frac{\tau_{0} N_{0}^{2}}{\kappa f^{2}} b^{2} \quad \mathscr{T}_{\text {frm }}=\frac{1}{2} \frac{N_{b}^{2}}{\kappa L f^{2}}\left(h^{2}-h_{b}^{2}\right) \mathscr{F}(-h)$.

The vertical scale $b$ characterizes the first moment of the local Brunt-Väisälä frequency, such that $b^{2} N_{0}^{2}=-\int z N^{2} \mathrm{~d} z$ and $b N_{0}^{2}=\int N^{2} \mathrm{~d} z$, and $N_{b}$ refers to some mean value in the deep layer. The parameter $h_{b}$ arises from the first moment of the bottom form stress in Eq. (18); it lies in the interval $D \leq h_{b} \leq h$ where $D$ is the minimum depth of topographic barriers on the zonal path. The balance of total momentum, Eq. (14), may be used to replace the bottom form stress $\mathscr{F}(-h)$ by $-L \tau_{0}$. Figure 11 displays the Eulerian contribution in the form

$\frac{\mathscr{T}_{0}+\mathscr{T}_{\text {frm }}}{\mathscr{T}_{0}}=1-\frac{1}{2} \frac{h^{2}-h_{b}^{2}}{b^{2}} \frac{N_{b}^{2}}{N_{0}^{2}}$

as function of $h_{b}$ and $N_{b} / N_{u}$ for the values appropriate to the POP experiment at $59^{\circ}$ latitude where $b=615 \mathrm{~m}$. The value of $N_{b} / N_{0}$ at this latitude is about 0.12 . Thus, to obtain a fraction of about 0.4 , as suggested for the ratio $\mathscr{T}_{\text {frm }} / \mathscr{T}_{0}$ in Table 1 , the form stress parameter $h_{b}$ must be in the range 1000 to $2000 \mathrm{~m}$.

The frictional terms

We assume that the subgrid stress $\tau$ is effective only in Ekman layers of thickness $\delta$ close to the surface and bottom. Then, assuming a linear decrease of the stress in these layers, we obtain:

$\mathscr{T}_{\mathrm{fr}} \approx \frac{\tau_{\mathrm{fr}} N_{\mathrm{fr}}^{2}}{\kappa f^{2}} \delta^{2}$,

where the index fr refers either to the frictional surface or bottom layer. Thus, $\mathscr{T}_{\mathrm{fr}}$ is very small compared to $\mathscr{T}_{0}$, of order $(\delta / b)^{2}$, and even if the bottom is flat and the wind stress must be balanced by the frictional stress at the bottom, i.e., $\tau_{\mathrm{fr}} \approx \tau_{0}$, the frictionally induced zonal transport, $\mathscr{T}_{\text {fr }}$, is negligible.

\section{The residual circulation term}

As mentioned above, the contribution from $\psi_{\text {res }}$,

$\mathscr{T}_{\text {res }}=-\frac{1}{\kappa f} \int_{-h}^{0} \mathrm{~d} z \frac{z N^{2}}{L} \psi_{\text {res }}$

describes the thermohaline forcing of the zonal mean current. We consider the solution (10) of $\psi_{\text {res }}$ in terms of the surface flux and interior conversion terms. Suppose now for simplicity that all isopycnals crossing the latitude $y=Y_{\mathrm{DP}}$ of Drake Passage - where Eq. (23) is considered - outcrop to the south (see Fig. 3 for POP) so that the condition $\Sigma\left(Y_{\mathrm{DP}}, z\right)=\Sigma^{m l}\left(y=y_{\Sigma}\right)$ maps the depth interval at $Y_{\mathrm{DP}}$ to the latitude band south of Drake Passage, resulting in $y=y_{\Sigma}\left(Y_{\mathrm{DP}}, z\right)$ or $z=z_{\Sigma}\left(Y_{\mathrm{DP}}, y\right)$. If we insert Eq. (10) into Eq. (23), two contributions to the ACC transport arise from thermohaline fluxes in the system, one from fluxes across the sea surface south of Drake Passage, and one from fluxes across the zonal mean isopycnals in the interior, also south of Drake Passage. The surface flux induces the amount

$\mathscr{T}_{\text {res }}^{\text {surf }}=\frac{1}{\kappa f} \int_{y_{\Sigma}\left(Y_{\mathrm{DP}},-h\right)}^{Y_{\mathrm{DP}}} z_{\Sigma}\left(Y_{\mathrm{DP}}, y^{\prime}\right) \frac{\mathscr{H}_{0}\left(y^{\prime}\right)}{L} \mathrm{~d} y^{\prime}$.

The surface density flux $\mathscr{H}_{0}(y)$ at the latitude $y$ is weighted in above mixed layer contribution by the depth $z_{\Sigma}$ at latitude $Y_{\mathrm{DP}}$ of the isopycnal that outcrops at $y$. A second term, arising from subsurface subgrid fluxes, vanishes for an adiabatic interior, and a third term comes from $J^{\perp}$ describing cross-isopycnal fluxes in a zonally integrated framework.

\section{References}

Andrews DG, Holton JR, Leovy CB (1987) Middle atmosphere dynamics. Academic Press, New York, p 489

Barnier B, Siefridt L, Marchesiello P (1995) Thermal forcing for a global ocean circulation model using a 3-year climatology. J Mar Syst 6: 363-380

Best SE, Ivchenko VO, Richards KJ, Smith RD, Malone RC (1999) Eddies in numerical models of the Antarctic Circumpolar Current and their influence on the mean flow. J Phys Oceanogr 29: $328-350$ 
Cai W, Baines P (1996) Interactions between thermohaline- and wind-driven circulations and their relevance to the dynamics of the Antarctic Circumpolar Current, in a coarse-resolution global ocean general circulation model. J Geophys Res 101: 14073-14093

Charney JG, DeVore JG (1979) Multiple-flow equilibra in the atmosphere and blocking. J Atmos Sciences 36: 1205-1216

Döös K, Webb DJ (1994) The Deacon cell and other meridional cells of the Southern Ocean. J Phys Oceanogr 24: 429-442

FRAM group (1991) An eddy-resolving model of the Southern Ocean. EOS 72(15): 169-175

Gent PR, Large WG, Bryan FO (2001) What sets the mean transport through Drake Passage? J Geophys Res 106: 26932712

Gille ST (1997) The Southern Ocean momentum balance: evidence for topographic effects from numerical model output and altimeter data. J Phys Oceanogr 27: 2219-2232

Gnanadesikan A, Hallberg RW (2000) On the relationship of the Circumpolar Current to Southern Hemisphere winds in coarseresolution ocean models. J Phys Oceanogr 30: 2013-2034

Green JSA (1970) Transfer properties of the large-scale eddies and the general circulation of the atmosphere. Quart J Roy Met Soc 96: $157-185$

Hughes C, Ash E (2001) Eddy forcing of the mean flow in the Southern Ocean. J Geophys Res 106: 2713-2722

Ivchenko VO, Richards KJ, Stevens DP (1996) The dynamics of the Antarctic Circumpolar Current. J Phys Oceanogr 26: 753774

Johnson GC, Bryden HL (1989) On the size of the Antarctic Circumpolar Current. Deep Sea Res 36: 39-53

Karoly DJ, McIntosh PC, Berrisford P, McDougall TJ (1997) Similarities of the Deacon cell in the Southern Ocean and Ferrel cells in the atmosphere. Quart J Roy Meteorol Soc 123: 519-526

Killworth P, Nanneh MM (1994) Isopycnal momentum budget of the Antarctic Circumpolar Current in the Fine Resolution Antarctic Model. J Phys Oceanogr 24: 1201-1223

Le Traon P-Y, Nadal F, Ducet N (1998) An improved mapping method of multisatellite altimeter data. J Atmos Ocean Technol 15: $522-534$

Levitus S (1982) Climatological atlas of the world ocean. National Oceanic and Atmospheric Administration. Tech Pap 3, p 173

Maltrud ME, Smith RD, Semtner AJ, Malone RC (1998) Global eddy-resolving ocean simulations driven by 1985-1994 atmospheric winds. J Geophys Res 103: 308251-30853
Marsh R, Nurser AJG, Megann AP, New AL (2000) Water mass transformation in the Southern Ocean of a global isopycnal coordinate GCM. J Phys Oceanogr 30: 1013-1045

Marshall J, Olbers D, Ross H, Wolf-Gladrow D (1993) Potential vorticity constraints on the dynamics and hydrography in the Southern Ocean. J Phys Oceanogr 23: 465-487

McIntosh PC, McDougall TJ (1996) Isopycnic averaging and the residual mean circulation. J Phys Oceanogr 26: 1656-1660

Olbers D (1998) Comment on "On the obscurantist physics of 'form drag' in theorizing about the Circumpolar Current". J Phys Oceanogr 28: 1647-1654

Olbers D, Wübber C (1991) The role of wind and buoyancy forcing of the Antarctic Circumpolar Current. In: Latif M (ed) Strategies for future climate research. Max-Planck-Institut für Meteorologie, Hamburg pp. 161-191

Olbers D, Völker C (1996) Steady states and variability in oceanic zonal flows. In: Anderson DLT, Willebrand J (eds) Decadal climate variability dynamics and predicition. Springer, Berlin, Heidelberg, New York, pp 407-443

Olbers D, Gouretski V, Seiß G, Schröter J (1992) Hydrographic atlas of the Southern Ocean. Alfred Wegener Institute for Polar and Marine Research, Bremerhaven, 17 pp, 82 plates

Rintoul SR, Hughes C, Olbers D (2001) The Antarctic Circumpolar Current system. In: Siedler G, Church J, Gould J (eds) Ocean circulation and climate. Academic Press, New York, pp 271-302

Smith RD, Dukowicz JK, Malone RC (1992) Parallel ocean general circulation modeling. Physica (D) 60: 38-61

Speer K, Rintoul S, Sloyan B (2000) The diabatic Deacon cell. J Phys Oceanogr 30: 3212-3222

Stevens D, Ivchenko VO (1997) The zonal momentum balance in an eddy-resolving general-circulation model of the Southern Ocean. Quart J Roy Meteor Soc 123: 929-951

Stone PH (1972) A simplified radiative-dynamical model for the static stability of rotaing atmospheres. J Atmos Sci 29: 405-418

Visbeck M, Marshall J, Haine T, Spall M (1997) Specification of eddy transfer coefficients in coarse-resolution ocean circulation models. J Phys Oceanogr 27: 381-402

Völker C (1999) Momentum balance in zonal flows and resonance of baroclinic Rossby waves. J Phys Oceanogr 29: 1666-1681

Wolff J-O, Maier-Reimer E, Olbers DJ (1991) Wind-driven flow over topography in a zonal $\beta$-plane channel: a quasi-geostrophic model of the Antarctic Circumpolar Current. J Phys Oceanogr 21(2): 236-264 